Repositorio Institucional de la Universidad Autónoma de Madrid

https://repositorio.uam.es

Esta es la versión de autor del artículo publicado en:

This is an author produced version of a paper published in:

PACIFIC JOURNAL OF MATHEMATICS 292.2 (2018): 427-478

DOI: http://doi.org/10.2140/pjm.2018.292.427

Copyright: @ 2018 MSP

El acceso a la versión del editor puede requerir la suscripción del recurso Access to the published version may require subscription 


\title{
Formal confluence of quantum differential operators
}

\author{
Bernard Le Stum \& Adolfo Quirós*
}

\begin{abstract}
We prove that a usual differential operator is formally the limit of quantum differential operators. For this purpose, we introduce the notion of twisted differential operator of infinite level and prove that, formally, such an object is independent of the choice of the twist. Our method provides explicit formulas.
\end{abstract}

\section{Contents}

\begin{tabular}{|ll|l}
\hline 1 & Twisted principal parts & 4
\end{tabular}

\begin{tabular}{lll}
\hline 2 & Twisted differential forms & 10
\end{tabular}

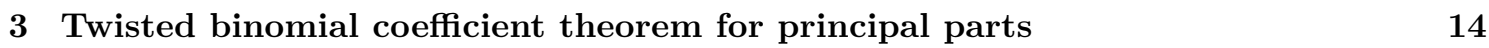

\begin{tabular}{lll}
\hline & Twisted differential operators of infinite level & 16
\end{tabular}

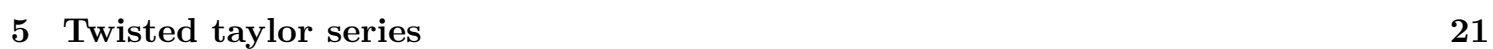

6 Quantum differential operators 24

\begin{tabular}{|ll|l}
\hline 7 & Formal deformations of twisted differential operators & 27
\end{tabular}

8 Formal confluence 33

$\begin{array}{|cc|}9 & \text { Formal confluence in positive quantum characteristic }\end{array} 36$

\begin{tabular}{ll}
\hline References & 40
\end{tabular}

\section{Introduction}

Classically, the finite difference operator and the $q$-difference operator are given by the formulas

$$
\Delta_{h}(f)(x)=\frac{f(x+h)-f(x)}{h} \text { and } \quad \delta_{q}(f)(x)=\frac{f(q x)-f(x)}{q x-x},
$$

*Quirós was supported by grant MTM2015-68524-P (MINECO/FEDER, Spain/EU). 
and we can obtain the differentiation operator by passing to the limit

$$
\partial(f)=\lim _{h \rightarrow 0} \Delta_{h}(f)=\lim _{q \rightarrow 1} \delta_{q}(f) .
$$

By using any of these operators, we may consider the notion of finite difference system, $q$-difference system or differential system. The confluence process consists in using the solutions of a difference (finite difference or $q$-difference) system in order to approximate the solutions of a differential system.

In the late 19th century, C. Guichard solved the problem of integration along a finite difference operator in [18]. In the early 20th century, in a couple of articles in the Transactions of the AMS ([9 and 6]), Georges D. Birkhoff and R. D. Carmichael gave reasonable conditions for solving linear finite difference systems. Shortly after, Carmichael applied the same methods to linear $q$ difference systems ([10]). This was later improved by C. Raymond Adams ([1]). There exists an abundant literature on these questions and we would like to jump to the present and mention the recent paper by Anne Duval and Julien Roques [15] where they study Fuchsian difference systems and their confluence. More generally, the school of Jean-Pierre Ramis produced a lot of interesting materials during the last two decades (see [12 for a survey). Among them, we can mention in particular the work of Jacques Sauloy on resolution, classification and confluence of Fuchsian differential equations (see [28] for example) and on the Galois theory of these equations ([29]).

While these works look at equations over the complex numbers, difference systems have also been studied over $p$-adic fields by Lucia di Vizio (see [13] for example) and Andrea Pulita ([26], [27]). Pulita showed that, over a $p$-adic field, confluence is a very rigid process in the sense that we can approximate a linear differential system by a $q$-difference system that has exactly the same (formal) solutions. Finally, we want to mention the work of Charlotte Hardouin ([19]) who studies $q$-difference Galois theory over a field of positive characteristic in the "exotic" case in which $q$ is a root of unity (which is also taken into account by Pulita).

Our approach here is very general in the sense that we do not make any assumption about the base field or the value of the parameter $q$ for example. Actually, differential systems and difference systems may be seen as particular instances of what we called twisted differential systems in 23] (see also [2 for a similar approach). Let us be more precise. If $R$ is a commutative ring, then a twisted $R$-algebra (or a difference $R$-algebra, if we name them in the spirit of [11]) is a commutative $R$-algebra $A$ endowed with an $R$-linear ring endomorphism $\sigma$. Actually, in 23 we allowed more generally families of endomorphisms satisfying some relations but we will concentrate here on the one dimensional case and we will use either one endomorphism or a system of roots of this endomorphism. One may then consider the $\sigma$-derivations $D$ of $A$, or more generally, the $\sigma$-derivations $D$ of an $A$-module $M$ : $R$-linear maps that satisfy the twisted Leibnitz rule

$$
D(x s)=D(x) s+\sigma(x) D(s) .
$$

A $\sigma$-differential module is then defined as an $A$-module $M$ endowed with an action by $\sigma$-derivations of all the $\sigma$-derivations of $A$. In the classical situation above, we had $\sigma(x)=x, \sigma(x)=q x$ or $\sigma(x)=x+h$, giving rise to an action of $\partial, \delta_{q}$ or $\Delta_{h}$ respectively. In our more general setting, the confluence question amounts to comparing twisted differential systems for different twists.

Our method is original in the sense that it relies on a generalization of the classical notion of ring of differential operators. In order to do that, we may first fix what we call a $\sigma$-coordinate $x \in A$, so that there exists a basis $\partial_{\sigma, A}$ for the $\sigma$-derivations of $A$ such that $\partial_{\sigma, A}(x)=1$. We may then define the twisted Weyl algebra $\mathrm{D}_{\sigma}$ as the Ore extension of $A$ by $\sigma$ and $\partial_{\sigma, A}$ (as in [8], proposition 1.4). This is the free $A$-module on the generators $\partial_{\sigma}^{k}$ for $k \in \mathbb{N}$ that satisfies

$$
\partial_{\sigma} \circ f=\partial_{\sigma, A}(f)+\sigma(f) \partial_{\sigma}
$$

when $f \in A$. A $\sigma$-differential module is then the same thing as a $\mathrm{D}_{\sigma}$-module. So now, what we want to understand is the relation between $\mathrm{D}_{\sigma}$ and $\mathrm{D}_{\tau}$ when $\tau$ is another $R$-linear endomorphism of $A$ with the same twisted coordinate $x$. In the end, we will be essentially interested in $\tau(x)=x$ 
(usual case and we will simply write $D$ instead of $D_{\tau}$ ) and $\sigma(x)=q x+h$ with $q, h \in R$ (quantum case). Note that the condition of $q$ being a root of unity in the quantum case below is analog to having a positive characteristic in the usual case and will require some care.

One may usually see a ring of differential operators as some dual of a ring of formal functions (see the first section of [17] for example). Doing this directly for $\mathrm{D}_{\sigma}$ would require to understand the notion of $\sigma$-divided powers on the function side. This is a very interesting question that we postpone to a forthcoming article. Here, we will actually replace the twisted Weyl Algebra $\mathrm{D}_{\sigma}$ with a Grothendieck ring of differential operators $\mathrm{D}_{\sigma}^{(\infty)}$ (so that the $\sigma$-divided powers live naturally on the differential operator side). It so happens that the classical construction works incredibly well for this particular generalization. It is actually sufficient to replace the usual powers of an ideal with the twisted powers introduced in [22]. But now we are faced with two questions: the comparison between $\mathrm{D}_{\sigma}^{(\infty)}$ and $\mathrm{D}_{\tau}^{(\infty)}$ on one hand and the comparison of $\mathrm{D}_{\sigma}$ with $\mathrm{D}_{\sigma}^{(\infty)}$ on the other.

We will show that, if we denote by $\widehat{D}_{\sigma}^{(\infty)}$ the completion along the divided powers of $\partial_{\sigma}$, then there always exists a natural isomorphism $\widehat{\mathrm{D}}_{\sigma}^{(\infty)} \simeq \widehat{\mathrm{D}}_{\tau}^{(\infty)}$ (the formal deformation of proposition 7.4 and we will be able to give very explicit formulas. For example, in the case $\tau(x)=x$ and $\sigma(x)=q x$, we can write (over a field of characteristic zero when $q$ is not a root of unity):

$$
\partial_{\sigma}=\sum_{k=1}^{\infty} \frac{1}{k !}(q-1)^{k-1} x^{k-1} \partial^{k} \quad \text { and } \quad \partial=\sum_{k=1}^{\infty} \frac{(1-q)^{k}}{1-q^{k}} x^{k-1} \partial_{\sigma}^{k} .
$$

In the quantum situation, there exists a canonical map $\mathrm{D}_{\sigma} \rightarrow \mathrm{D}_{\sigma}^{(\infty)}$ whose image is exactly the ring $\overline{\mathrm{D}}_{\sigma}$ of small (or naive) differential operators. Under some conditions on $q$ ( $R q$-divisible and $q-\operatorname{char}(R)=0$, see [23]), then all three rings $\mathrm{D}_{\sigma}, \overline{\mathrm{D}}_{\sigma}$ and $\mathrm{D}_{\sigma}^{(\infty)}$ are actually equal. This happens for example in the usual case when $R$ is a $\mathbb{Q}$-algebra and in the quantum case when $q$ belongs to a subfield $K$ of $R$ and $q$ is not a root of unity. When all these conditions are satisfied, we easily derive from the formal deformation our first confluence theorem 8.3 there exists a map $\mathrm{D}_{\sigma} \rightarrow \widehat{\mathrm{D}}$ with dense image.

We will also consider the case where $q$ is a primitive $p$-th root of unity (but still $R$ a $\mathbb{Q}$-algebra). It is then necessary to use a complete family $q$ of $p^{n}$-th roots of $q$. We can define what we call a rooted Weyl algebra $\mathrm{D}_{\underline{\sigma}}$ by taking the limit on all $\mathrm{D}_{\sigma_{n}}$ and build a map $\mathrm{D}_{\underline{\sigma}} \rightarrow \widehat{\mathrm{D}}$ with dense image. This is the second confluence theorem 9.13

It is interesting to notice that this last theorem puts together the ring $D$ that supports the conjecture of Jacques Dixmier [14] (whose higher version is equivalent to the Jacobian conjecture [5]) and an Azumaya algebra where the Morita equivalence could be applied. Recall that the Jacobian conjecture (or global inversion theorem) states that an endomorphism of the complex affine space whose Jacobian is invertible is necessarily an isomorphism. Recall also that Dixmier's conjecture states that any endomorphism of a complex Weyl algebra such as $D$ is always an automorphism. This conjecture is not valid for $D_{\underline{\sigma}}$. However, this last ring is a lot easier to study because it is essentially a matrix algebra.

In a forthcoming paper, we will prove an ultrametric version of the results presented here. More precisely, we will introduce the notion of twisted differential operator of given radius on an affinoid algebra and show that this notion is essentially independent of the choice of the twist. This will lead to an explicit equivalence between differential systems and difference systems, generalizing a theorem of Pulita in [26]. Recall that the first results in this direction were already obtained by Yves André and Lucia Di Vizio in [3]).

In a forthcoming joint paper with Michel Gros, we will introduce the notion of quantum divided power and apply our methods in order to obtain a ring of quantum differential operators of level zero. It happens to be isomorphic to the quantum Weyl algebra. However, this new approach also provides the notion of quantum $p$-curvature. We will then use some Frobenius action and obtain an Azumaya splitting of the quantum Weyl algebra as well as a quantum Simpson's correspondence much as in [16]. 
Both authors thank Michel Gros for all the fruitful conversations that we had all together.

For us, a ring has an associative multiplication (not commutative in general) and a two-sided unit. Morphisms of rings are always assumed to preserve the unit. A module always means a left module. We will essentially consider 1-twisted rings from [23] and simply call them twisted rings. More precisely, throughout the paper, $R$ will denote a commutative ring and $A$ a twisted commutative $R$-algebra: a commutative $R$-algebra endowed with an $R$-linear ring endomorphism $\sigma_{A}$.

\section{Twisted principal parts}

We will introduce here the notion of twisted principal part (functions on twisted infinitesimal neighborhoods of the diagonal).

We will begin by ignoring the endomorphism $\sigma_{A}$ and concentrating on the commutative $R$-algebra $A$. The tensor product $P_{A / R}:=A \otimes_{R} A$ has two $A$-algebra structures, one coming from the action on the left and the other one coming from the action on the right. Unless otherwise specified, we will use the left structure when we see $P_{A / R}$ as an $A$-module. However, when $M$ is an $A$-module, the notation $P \otimes_{A} M$ will implicitly mean that we use the action of $A$ on the right to build the tensor product and that the resulting object will be seen as an $A$-module using the action of $A$ on the left.

In practice, we will write $x:=x \otimes 1 \in P_{A / R}$ and $\tilde{x}:=1 \otimes x \in P_{A / R}$. In other words, with these notations, the action on the left is multiplication by $x$ and the action on the right is multiplication by $\tilde{x}$. Any element of $P_{A / R}$ can be written as a finite sum $\sum x_{i} \tilde{y}_{i}$. At some point, we will call Taylor map the embedding on the right and denote it by

$$
\begin{aligned}
\theta_{A / R}: \quad & A \longrightarrow P_{A / R} . \\
x & \longmapsto \tilde{x}
\end{aligned}
$$

We will then call $\tilde{x}=\theta_{A / R}(x)$ the Taylor expansion of $x \in A$ (more on this vocabulary later on).

We will also consider the canonical map corresponding to the projection that forgets the middle term:

$$
\begin{gathered}
A \otimes_{R} A \stackrel{\delta_{A / R}}{\longrightarrow} A \otimes_{R} A \otimes_{R} A . \\
x \otimes y \longmapsto \\
\longrightarrow \otimes 1 \otimes y
\end{gathered}
$$

It is a morphism of $R$-algebras that may also be seen as a map

$$
\begin{aligned}
& P_{A / R} \stackrel{\delta_{A / R}}{\longrightarrow} P_{A / R} \otimes_{A} P_{A / R}, \\
& x \tilde{y} \longmapsto x \otimes \tilde{y}
\end{aligned}
$$

where $A$ acts on the right on the first factor and on the left on the second one in the tensor product.

Let $I_{A / R}$ be the kernel of the multiplication morphism

$$
\begin{aligned}
P_{A / R} \longrightarrow A \\
x \tilde{y} \longrightarrow x y
\end{aligned}
$$

(that corresponds to the diagonal embedding). The ideal $I_{A / R}$ is generated by the image of the linear map

$$
\begin{aligned}
& A \stackrel{d_{A / R}}{\longrightarrow} P_{A / R} \\
& x \longmapsto \xi:=\tilde{x}-x
\end{aligned}
$$

(that corresponds to the difference between the projections). In practice, we will usually drop the index $A / R$ and simply write $P, I, \theta, \delta$ and $d$.

Since we will make regular use of linearization, we introduce it formally now: 
Definition 1.1. Let $M, N$ be two $A$-modules and $u: M \rightarrow N$ an $R$-linear map. Then, the A-linearization of $u$ is the $A$-linear map

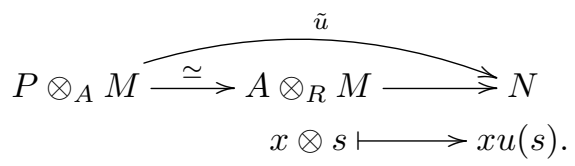

Recall that, in the tensor product, we use the action of the right on $P$. Therefore, we have

$$
\forall x, y \in A, \forall s \in M, \quad \tilde{u}(x \tilde{y} \otimes s)=x u(y s) .
$$

As an example, we see that the multiplication map $P \rightarrow A$ is the $A$-linearization of the identity of $A$, seen as an $R$-linear map.

For further use, we also mention the following result, that follows immediately from the definitions:

Lemma 1.2. If we are given two $R$-linear maps $\varphi: M \rightarrow N$ and $\psi: L \rightarrow M$, then the linearization of $\varphi \circ \psi$ factors as

$$
P \otimes_{A} L \stackrel{\delta \otimes \operatorname{Id}}{\longrightarrow} P \otimes_{A} P \otimes_{A} L \stackrel{\operatorname{Id} \otimes_{A} \tilde{\psi}}{\longrightarrow} P \otimes_{A} M \stackrel{\tilde{\varphi}}{\longrightarrow} N
$$

Now we make the endomorphism $\sigma_{A}$ enter the game. We will consider $P:=A \otimes_{R} A$ as a twisted $R$ algebra by using $\sigma_{A}$ on the left and the identity the right: in other words, we set $\sigma_{P}:=\sigma_{A} \otimes_{R} \operatorname{Id}_{A}$. Alternatively, this is the unique structure of twisted $R$-algebra on $P$ such that

$$
\forall x \in A, \quad \sigma_{P}(x)=\sigma_{A}(x) \quad \text { and } \quad \sigma_{P}(\tilde{x})=\tilde{x} .
$$

In particular, we may also consider $P$ as a twisted $A$-algebra, in the sense that $P$ is endowed with a $\sigma_{A}$-linear ring endomorphism $\sigma_{P}$. We will often drop the indexes $A$ and $P$ and simply write $\sigma$ for both maps (so that $\sigma(\tilde{x})=\tilde{x}$ ) when there is no ambiguity.

Before we do anything else, let us prove the following result, which is quite elementary, but very useful:

Lemma 1.3. If $x \in A$ and $\xi:=\tilde{x}-x \in P_{A / R}$, then,

$$
\sigma_{P}(\xi)=\xi+y \quad \text { with } \quad y:=x-\sigma_{A}(x)
$$

Proof We have

$$
\sigma(\xi)=\sigma(\tilde{x}-x)=\sigma(\tilde{x})-\sigma(x)=\tilde{x}-\sigma(x)=\tilde{x}-x+x-\sigma(x)=\xi+y .
$$

Lemma 1.4. The kernel of the A-linearization

$$
\begin{aligned}
& P_{A / R} \stackrel{\tilde{\sigma}_{A}}{\longrightarrow} A \\
& x \tilde{y} \longmapsto \\
& \longrightarrow \sigma_{A}(y)
\end{aligned}
$$

of $\sigma_{A}$ is $\sigma_{P}\left(I_{A / R}\right)$.

Proof Recall that $\sigma(I)$ denotes the ideal generated by the image of $I$. As a consequence, since $I$ is generated by the image of $d$, we see that ker $\tilde{\sigma}$ will be generated by the image of

$$
\begin{aligned}
& A \stackrel{\sigma \circ d}{\longrightarrow} P \\
& x \longmapsto \tilde{x}-\sigma(x)
\end{aligned} .
$$

Now, we have

$$
\forall x \in A, \quad \tilde{\sigma}(\tilde{x}-\sigma(x))=\sigma(x)-\sigma(x)=0,
$$


and it follows that $\sigma(I) \subset \operatorname{ker} \tilde{\sigma}$. Conversely, by definition, we have

$$
\forall x \in A, \quad \sigma(x) \equiv \tilde{x} \quad \bmod \sigma(I) .
$$

Therefore, if $f:=\sum x_{i} \tilde{y}_{i} \in \operatorname{ker} \tilde{\sigma}$, we have

$$
f \equiv \sum x_{i} \sigma\left(y_{i}\right)=\tilde{\sigma}(f)=0 \bmod \sigma(I)
$$

and we see that ker $\tilde{\sigma} \subset \sigma(I)$.

Remarks 1. It is sometimes convenient to use the bimodule language. An A-sesquimodule $M$ is an $A$-bimodule such that

$$
\forall x \in A, s \in M, \quad \sigma_{A}(x) \cdot s=s \cdot x .
$$

Note that we are using the reverse convention from André's in 22 so that forgetting the right action induces an equivalence (an isomorphism) between $A$-sesquimodules and left $A$-modules (we will use this identification).

2. The $R$-algebra $P$ has a canonical $A$-bimodule structure which is completely independent of the choice of $\sigma_{A}$. If we endow $A$ with its sesquimodule structure, then the linearization $\tilde{\sigma}$ of $\sigma_{A}$ is a morphism of $A$-bimodules: we always have

$$
\forall x, y \in A, f \in P, \quad \tilde{\sigma}(x \cdot f \cdot y)=\tilde{\sigma}(x f \tilde{y})=x \tilde{\sigma}(f) \sigma(y)=x \cdot \tilde{\sigma}(f) \cdot y .
$$

It follows that ker $\tilde{\sigma}$ has a natural $A$-bimodule structure. Actually, since $\tilde{\sigma}$ is a ring homomorphism, then ker $\tilde{\sigma}$ is an ideal and therefore automatically an $A$-bimodule.

Recall from 22 that the twisted powers of $I$ are

$$
I^{(0)}=P, \quad I^{(1)}=I, \quad I^{(2)}:=I \sigma(I), \quad \ldots, \quad I^{(n)}:=I \sigma(I) \cdots \sigma^{n-1}(I),
$$

where images and products of ideals are meant as ideals. We will write $I_{A / R}^{(n)_{\sigma}}$ when we want to make clear the dependence on $\sigma$ and $A / R$.

Definition 1.5. The $A$-module of twisted principal parts of order $n \in \mathbb{N}$ (and infinite level) of $A$ is

$$
P_{A / R,(n)_{\sigma}}:=P_{A / R} / I_{A / R}^{(n+1)_{\sigma}} .
$$

The A-module of twisted principal parts of infinite order (and infinite level) of $A$ is the twisted completion:

$$
\widehat{P}_{A / R, \sigma}:=\varliminf_{\varlimsup} P_{A / R,(n)_{\sigma}} .
$$

Note that these $A$-modules all have natural $R$-algebra structures and that the definition also makes sense for $n=-1$ so that $P_{A / R,(-1)_{\sigma}}=0$. Again, we will often drop the indexes $A / R$ when we believe that there is no risk of confusion and simply write $P_{(n)_{\sigma}}$ and $\widehat{P}_{\sigma}$. We will also drop the index $\sigma$ when $\sigma_{A}=\operatorname{Id}_{A}$.

Examples 1. When $A$ is trivially twisted, which means that $\sigma_{A}:=\operatorname{Id}_{A}$, this notion of principal parts coincides with the usual one (definition 16.3 .1 of [17]), and therefore many of the basic objects we will construct are twisted versions of those in [17.

2. When $A=R[x]$, we have $P=R[x, \xi]$ with $\xi=\tilde{x}-x$ and $I=(\xi)$. Moreover, $\sigma(\xi)=\xi+y$ with $y=x-\sigma(x)$. It follows that

$$
\sigma^{n}(\xi)=\xi+(n)_{\sigma}(y)=\xi+x-\sigma^{n}(x),
$$

with $(n)_{\sigma}:=1+\cdots+\sigma^{n-1}$. Therefore, we have

$$
P_{(n)}=R[x, \xi] / \prod_{i=0}^{n}\left(\xi+(i)_{\sigma}(y)\right)=R[x, \xi] / \prod_{i=0}^{n}\left(\xi+x-\sigma^{n}(x)\right) .
$$


(a) In the case $\sigma(x)=x$, we get $P_{(n)}=R[x, \xi] / \xi^{n+1}$ as expected.

(b) More generally, if we assume that $\sigma(x)=x+h$ with $h \in R$, we obtain

$$
P_{(n)}=R[x, \xi] / \prod_{i=0}^{n}(\xi-i h) .
$$

(c) On the other hand, if we let $\sigma(x)=q x$ with $q \in R$, we find

$$
P_{(n)}=R[x, \xi] / \prod_{i=0}^{n}\left(\xi+\left(1-q^{i}\right) x\right) .
$$

When $R \rightarrow R^{\prime}$ is a homomorphism of commutative rings, we endow $A^{\prime}:=R^{\prime} \otimes_{R} A$ with $\sigma_{A^{\prime}}:=\operatorname{Id}_{R^{\prime}} \otimes_{R} \sigma_{A}$.

Proposition 1.6. Let $R \rightarrow R^{\prime}$ be a homomorphism of commutative rings and $A^{\prime}:=R^{\prime} \otimes_{R} A$. Then, we have for all $n \in \mathbb{N}$,

$$
A^{\prime} \otimes_{A} P_{A / R,(n)_{\sigma}} \simeq P_{A^{\prime} / R^{\prime},(n)_{\sigma}} .
$$

Proof If we let $P^{\prime}:=P_{A^{\prime} / R^{\prime}}$, then there exists an canonical isomorphism $A^{\prime} \otimes_{A} P \simeq R^{\prime} \otimes_{R} P \simeq P^{\prime}$. Moreover, if we denote by $I^{\prime}$ the kernel of the multiplication morphism on $P^{\prime}$, we have $A^{\prime} \otimes_{A} I \simeq I^{\prime}$. And finally, $\operatorname{Id}_{A} \otimes_{A} \sigma_{P}$ corresponds to $\sigma_{P^{\prime}}$ under this isomorphism. Our assertion is therefore an immediate consequence of right exactness of tensor product.

Recall from 222 that a twisted $A$-algebra is an $A$-algebra $B$ endowed with a $\sigma_{A}$-linear ring endomorphism $\sigma_{B}$ (which simply means that $\forall f \in A, \sigma_{B}\left(f 1_{B}\right)=\sigma_{A}(f)$ ).

Proposition 1.7. If $B$ is a twisted commutative A-algebra, then there exists a canonical morphism of B-algebras

$$
B \otimes_{A} P_{A / R,(n)_{\sigma}} \rightarrow P_{B / R,(n)_{\sigma}} .
$$

When $B$ is a quotient (resp. a localization) of $A$ this map is surjective (resp. bijective).

Recall from Definition 1.7 of 23 that we call such a $B$ a twisted quotient (resp. twisted localization) of $A$.

Proof The morphism of twisted $R$-algebras $A \rightarrow B$ extends naturally to a morphism of twisted $R$-algebras $P_{A} \rightarrow P_{B}$. Since $I_{A}$ is sent into $I_{B}$, we see that, for all $n \in \mathbb{N}, \sigma^{n}\left(I_{A}\right)$ is sent into $\sigma^{n}\left(I_{B}\right)$ and the first assertion formally follows. In the case of a quotient map, all the maps involved are surjective.

Now, if $B:=S^{-1} A$ is a twisted localization of $A$, then $P_{B}$ is the localization of $P_{A}$ with respect to the monoid $S^{\prime}$ generated by $S$ and $\tilde{S}$, and we have $I_{B}=P_{B} \otimes_{P_{A}} I_{A}$. It immediately follows that for all $n \in \mathbb{N}$, we have $\sigma^{n}\left(I_{B}\right)=P_{B} \otimes_{P_{A}} \sigma^{n}\left(I_{A}\right)$, and therefore $I_{B}^{(n)}=P_{B} \otimes_{P_{A}} I_{A}^{(n)}$. Thus we see that

$$
P_{B,(n)_{\sigma}}=P_{B} /\left(P_{B} \otimes_{P_{A}} I_{A}^{(n+1)}\right)=P_{B} \otimes_{P_{A}} P_{A,(n)}=B \otimes_{A} P_{A,(n)} \otimes_{A} B .
$$

We need to remove the $B$ on the right hand side and it is sufficient to show that $\tilde{x}$ is invertible in $B \otimes_{A} P_{A,(n)}$ whenever $x \in S$. But we have

$$
\prod_{i=0}^{n}\left(\tilde{x}-\sigma^{i}(x)\right)=\prod_{i=0}^{n} \sigma^{i}(\tilde{x}-x) \in I_{A}^{(n+1)}
$$

from which we derive that there exists $f \in P_{A}$ such that

$$
f \tilde{x} \equiv \prod_{i=0}^{n} \sigma^{i}(x) \bmod I_{A}^{(n+1)} .
$$

Since $B=S^{-1} A$, we must have $\sigma(S) \subset B^{\times}$and it follows that $\prod_{i=0}^{n} \sigma^{i}(x) \in B^{\times}$. Thus, we see that $f \tilde{x}$ is invertible in $B \otimes_{A} P_{A,(n)}$ and it follows that $\tilde{x}$ is invertible too. 
As an illustration, we can give explicit formulas in the quantum situation. Recall that we introduced in $[22$ the notion of twisted powers of an element in a twisted ring. In particular, for $f \in P$, we will have

$$
f^{(0)}:=1, \quad f^{(1)}:=f, \quad f^{(2)}=f \sigma(f), \quad \ldots, \quad f^{(n+1)}=f \sigma(f) \cdots \sigma^{n}(f) .
$$

Recall also that the quantum binomial coefficients are defined by induction (see [22] for example) as

$$
\left(\begin{array}{l}
n \\
k
\end{array}\right)_{q}:=\left(\begin{array}{l}
n-1 \\
k-1
\end{array}\right)_{q}+q^{k}\left(\begin{array}{c}
n-1 \\
k
\end{array}\right)_{q}
$$

Proposition 1.8. Assume $\sigma(x)=q x$ with $q \in R$ and let $\xi=\tilde{x}-x$. Then, we have

$$
\forall n \in \mathbb{N}, \quad \xi^{(n)}=\sum_{j=0}^{n}(-1)^{j}\left(\begin{array}{l}
n \\
j
\end{array}\right)_{q} q^{\frac{j(j-1)}{2}} x^{j} \tilde{x}^{n-j}
$$

and

$$
\forall n \in \mathbb{N}, \quad \tilde{x}^{n}=\sum_{i=0}^{n}\left(\begin{array}{l}
n \\
i
\end{array}\right)_{q} x^{i} \xi^{(n-i)}
$$

Proof The first equality is essentially the quantum binomial formula (see proposition 2.14 in [22]):

$$
(\tilde{x}-x)^{(n)}=\sum_{j=0}^{n}\left(\begin{array}{l}
n \\
j
\end{array}\right)_{q}(-x)^{(j)} \tilde{x}^{(n-j)}
$$

For the second one, we compute the right hand side with the help of formula [1.1):

$$
\begin{aligned}
\sum_{i=0}^{n}\left(\begin{array}{l}
n \\
i
\end{array}\right)_{q} x^{i} \xi^{(n-i)} & =\sum_{i=0}^{n}\left(\begin{array}{c}
n \\
i
\end{array}\right)_{q} x^{i}\left(\sum_{j=0}^{n-i}(-1)^{j}\left(\begin{array}{c}
n-i \\
j
\end{array}\right)_{q} q^{\frac{j(j-1)}{2}} x^{j} \tilde{x}^{n-i-j}\right) \\
& =\sum_{k=0}^{n}\left(\sum_{i=0}^{k}\left(\begin{array}{c}
n \\
i
\end{array}\right)_{q}\left(\begin{array}{c}
n-i \\
k-i
\end{array}\right)_{q}(-1)^{k-i} q^{\frac{(k-i)(k-i-1)}{2}}\right) x^{k} \tilde{x}^{n-k}
\end{aligned}
$$

after rewriting $i+j=k$. Now, we have (using corollaries 2.7 and 2.8 in [22], for example)

$$
\sum_{i=0}^{k}\left(\begin{array}{l}
n \\
i
\end{array}\right)_{q}\left(\begin{array}{l}
n-i \\
k-i
\end{array}\right)_{q}(-1)^{k-i} q^{\frac{(k-i)(k-i-1)}{2}}=\left(\begin{array}{l}
n \\
k
\end{array}\right) \sum_{q}^{k}\left(\begin{array}{l}
k \\
i
\end{array}\right)_{q}(-1)^{k-i} q^{\frac{(k-i)(k-i-1)}{2}} .
$$

But the quantum binomial formula again implies that for $k>0$, we have

$$
\sum_{i=0}^{k}\left(\begin{array}{l}
k \\
i
\end{array}\right)_{q}(-1)^{k-i} q^{\frac{(k-i)(k-i-1)}{2}}=\prod_{i=0}^{k-1}\left(1-q^{i}\right)=0 .
$$

And it follows that

$$
\sum_{i=0}^{n}\left(\begin{array}{c}
n \\
i
\end{array}\right)_{q} x^{i} \xi^{(n-i)}=\tilde{x}^{n}
$$

as asserted.

We will need a slightly stronger notion of coordinate than the one used in [23]:

Definition 1.9. Let $x \in A$ and $\xi:=\tilde{x}-x \in P_{A / R}$.

1. Then, $x$ is a twisted coordinate for the twisted $R$-algebra $A$ or a $\sigma$-coordinate for the $R$ algebra $A$ if for all $n \in \mathbb{N}, P_{A / R,(n)_{\sigma}}$ is freely generated as an $A$-module by the images of $1, \xi, \xi^{2}, \ldots, \xi^{n}$. 
2. If $x$ is a twisted coordinate such that $\sigma(x)=q x+h$ with $q, h \in R$, then we will call it a quantum coordinate or q-coordinate and call $A$ a quantum $R$-algebra or $q$-R-algebra.

Examples 1. When $\sigma_{A}=\operatorname{Id}_{A}$, a twisted coordinate will be called a usual coordinate. In the case $A / R$ is smooth (of pure relative dimension one), then a usual coordinate is nothing but an étale coordinate: it means that the map

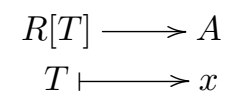

is étale.

2. If $A=R[x]$, then $x$ is always a twisted coordinate, whatever $\sigma$ is.

Proposition 1.10. 1. If $R \rightarrow R^{\prime}$ is a homomorphism of commutative rings and $x$ is a twisted coordinate on $A$, then $x$ becomes a twisted coordinate on $A^{\prime}:=R^{\prime} \otimes_{R} A$ (relatively to $R^{\prime}$ ).

2. If $B$ is a twisted localization of $A$ and $x$ is a twisted coordinate on $A$, then $x$ becomes a twisted coordinate on $B$.

Proof Follows from propositions 1.6 and 1.7

Remark Assume $A$ is a quantum $R$-algebra so that there exists a twisted coordinate $x$ on $A$ and $q, h \in R$ such that $\sigma(x)=q x+h$. Let us still denote by the same letter $x$ an indeterminate over $R$ and by $\sigma$ again the endomorphism of $R[x]$ given by the same formula. Then, $A$ becomes an $R[x]$-twisted algebra and we have a canonical isomorphism (free $A$-modules of the same rank with the same generators):

$$
A \otimes_{R[x]} P_{R[x] / R,(n)_{\sigma}} \simeq P_{A / R,(n)_{\sigma}} .
$$

In the next statement, we use the letter $\xi$ as an indeterminate over $A$ so that $A[\xi]$ denotes the polynomial ring and $A[\xi]_{\leq n}$ the submodule of polynomial of degree at most $n$. Ultimately, this should not create any confusion due to corollary 1.12 below.

Proposition 1.11. Let $x \in A$ and $y:=x-\sigma(x)$. We endow $A[\xi]$ with the unique $\sigma_{A}$-linear endomorphism such that $\sigma(\xi)=\xi+y$. Then, $x$ is a twisted coordinate on $A$ if and only if the morphism of twisted algebras

$$
\begin{gathered}
\phi: A[\xi] \longrightarrow P_{A / R} \\
\xi \longmapsto
\end{gathered}
$$

induces for all $n \in \mathbb{N}$, an isomorphism of A-algebras $A[\xi] / \xi^{(n+1)} \simeq P_{A / R,(n)_{\sigma}}$.

Proof First of all, it follows from lemma 1.3 that there exists such a morphism for all $n \in \mathbb{N}$. On the other hand,

$$
\xi^{(n+1)}=\prod_{0}^{n}\left(\xi+\left(x-\sigma^{i}(x)\right)\right)
$$

is a monic polynomial of degree $n+1$. Then, euclidean division tells us that the composite map

$$
A[\xi]_{\leq n} \longrightarrow A[\xi] \longrightarrow A[\xi] / \xi^{(n+1)}
$$

is an isomorphism of $A$-modules. Therefore the condition on $\phi$ is equivalent to the fact that the map

$$
\begin{aligned}
A[\xi]_{\leq n} & \longrightarrow P_{(n)_{\sigma}} \\
\xi \longmapsto & \longrightarrow \tilde{x}-x
\end{aligned}
$$

is bijective. And this exactly means that $P_{(n)_{\sigma}}$ is freely generated by the $n+1$ first powers of the images of $\tilde{x}-x$. 
When the polynomial ring $A[\xi]$ is endowed with a structure of $\sigma_{A}$-algebra, we will set

$$
A[[\xi]]_{\sigma}:=\lim A[\xi] / \xi^{(n+1)} .
$$

Corollary 1.12. With the same hypothesis, $x$ is a twisted coordinate on $A$ if and only if there exists an isomorphism of A-algebras

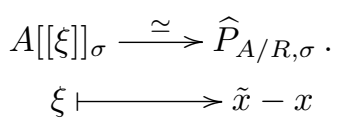

Corollary 1.13. Let $x \in A$ and $\xi:=\tilde{x}-x \in P$. Then, the following conditions are equivalent:

1. $x$ is a twisted coordinate on $A$

2. for all $n \in \mathbb{N}$, the $A$-module $P_{(n)_{\sigma}}$ is freely generated by the images of $1, \xi, \xi^{(2)}, \ldots, \xi^{(n)}$

3. for all $n \in \mathbb{N}$, the $A$-module $I^{(n)} / I^{(n+1)}$ is free of rank one on the image of $\xi^{(n)}$.

Corollary 1.14. If $A$ is a twisted localization of $R[x]$, then $x$ is a twisted coordinate on $A$.

Proof Using the second part of proposition 1.10 , we may assume that $A=R[x]$ in which case, this is a trivial consequence of proposition 1.11

\section{Twisted differential forms}

In this section, we study the module of twisted differential forms (of degree one) and make the link with twisted derivations. We use same notations as before.

Definition 2.1. The $A$-module of twisted differential forms on $A / R$ is

$$
\Omega_{A / R, \sigma}^{1}:=I_{A / R} / I_{A / R}^{(2)_{\sigma}} .
$$

Again, we will often drop the index $A / R$. Since we implicitly endow $P$ with the action of $A$ on the left, we will also always see $\Omega_{\sigma}^{1}$ as an $A$-module through the action on the left.

Examples 1. When $A$ is trivially twisted, then $\Omega_{\sigma}^{1}=I / I^{2}$ is the usual module of differential forms of $A$ over $R$.

2. If $A=R[x]$ is endowed with any $R$-algebra endomorphism $\sigma$, then $\Omega_{\sigma}^{1}$ is free of rank 1: with the notations of lemma 1.3 (so that $y=x-\sigma(x)$ ), we have

$$
\Omega_{\sigma}^{1} \simeq \xi R[x, \xi] / \xi(\xi+y) \simeq R[x, \xi] /(\xi+y) \simeq R[x] .
$$

Remarks 1 . Clearly, $\Omega_{\sigma}^{1}$ has a natural $A$-bimodule structure as a quotient of two ideals of $P$. It happens that this is identical to its $A$-sesquimodule structure: by definition, if $x \in A$, then $\tilde{x} \equiv \sigma(x) \bmod \sigma(I)$ and it follows that

$$
\forall f \in I, \quad f \tilde{x} \equiv f \sigma(x) \quad \bmod I \sigma(I) .
$$

2. The $\Omega_{\sigma}^{1}$ that appears in proposition 1.4.2.1 of [2] is exactly the same as ours (André calls $k$ what we call $R$ ).

3. Formula 2.1 is exactly the first step of the braiding described by Max Karoubi and Mariano Suárez-Álvarez in [21. 
4. One can define more generally the twisted de Rham complex $\Omega_{\sigma}^{\bullet}$ of $A$ as the quotient of the non commutative tensor algebra of $I$ by the graded differential ideal generated by $I \sigma(I)$. We will not consider this complex here.

Proposition 2.2. There exists a split exact sequence

$$
0 \longrightarrow \Omega_{A / R, \sigma}^{1} \longrightarrow P_{A / R,(1)_{\sigma}} \stackrel{\tilde{\sigma}_{A}}{\longrightarrow} A \longrightarrow 0
$$

Proof There exists such an exact sequence by definition of $P_{(1)_{\sigma}}$ and $\Omega_{\sigma}^{1}$. The $A$-module structure of $P$ provides a section of $\widetilde{\sigma}$.

Proposition 2.3. 1. If $R \rightarrow R^{\prime}$ is a homomorphism of commutative rings and $A^{\prime}:=R^{\prime} \otimes_{R} A$, then there exists an isomorphism

$$
A^{\prime} \otimes_{A} \Omega_{A / R, \sigma}^{1} \simeq \Omega_{A^{\prime} / R^{\prime}, \sigma}^{1}
$$

2. If $B$ is a twisted commutative A-algebra, then there exists a canonical B-linear map

$$
B \otimes_{A} \Omega_{A / R, \sigma}^{1} \rightarrow \Omega_{B / R, \sigma}^{1} .
$$

When $B$ is a quotient (resp. a localization) of $A$ this map is surjective (resp. bijective).

Proof Using proposition 2.2 this follows from propositions 1.6 and 1.7

Remark This last result does not hold however if we only require $A \rightarrow B$ to be an étale map (and not a localization map) as the following example shows. Let $R$ be any field of characteristic different from $2, A:=R[x]$ with $\sigma_{A}:=\operatorname{Id}_{A}$ and $B:=R\left[x, x^{-1}\right]$ with $\sigma_{B}(x):=-x$. Then the morphism $x \mapsto x^{2}$ is an étale twisted morphism but the morphism $(2.2)$ is the zero map. More precisely, if $\xi=\tilde{x}-x$, we have

$$
B \otimes_{A} \Omega_{A, \sigma}^{1}=(\xi) /\left(\xi^{2}\right) \text { and } \Omega_{B, \sigma}^{1}=(\xi) /\left(\xi^{2}+2 x \xi\right),
$$

where the ideals are taken inside $R\left[x, x^{-1}, \xi\right]$, and morphism 2.2 is induced by $\xi \mapsto \xi^{2}+2 x \xi$.

Recall from [23] that a twisted derivation of $A$ is an $R$-linear map into an $A$-module $M$ that satisfies the twisted Leibnitz rule:

$$
\forall x, y \in A, \quad D(x y)=x D(y)+\sigma(y) D(x)
$$

They form an $A$-module $\operatorname{Der}_{R, \sigma}(A, M)$.

Proposition 2.4. The canonical map $A \rightarrow \Omega_{A / R, \sigma}^{1}$ induced by $d$ is a twisted derivation. It provides us with a natural isomorphism

$$
\begin{array}{r}
\operatorname{Hom}_{A}\left(\Omega_{A / R, \sigma}^{1}, M\right) \stackrel{\simeq}{\longrightarrow} \operatorname{Der}_{R, \sigma}(A, M) \\
u \longmapsto D:=u \circ d
\end{array}
$$

whenever $M$ is an A-module.

In the future, we will also denote by $d: A \rightarrow \Omega_{\sigma}^{1}$ this universal twisted derivation when there is no risk of confusion.

Proof Using formula 2.1, we see that, inside $P$, we have

$$
\begin{aligned}
y d(x)+\sigma(x) d(y) & =y(\tilde{x}-x)+\sigma(x)(\tilde{y}-y) \\
& \equiv y(\tilde{x}-x)+\tilde{x}(\tilde{y}-y)=\widetilde{x y}-x y=d(x y) \quad \bmod I \sigma(I) .
\end{aligned}
$$


It follows that the induced map $d: A \rightarrow \Omega_{\sigma}^{1}$ is indeed a twisted derivation. This also implies that the map in $(2.3)$ is well defined. And it is clearly injective because $I$ is generated by the image of $d$.

We now show that it is surjective. If $D$ is a twisted derivation of $M$, we can consider its linearization

$$
\begin{aligned}
& P \stackrel{\tilde{D}}{\longrightarrow} M \\
& x \tilde{y} \longmapsto
\end{aligned} .
$$

By definition, the ideal $I \sigma(I)$ is generated by elements of the form

$$
f=(\tilde{x}-x)(\tilde{y}-\sigma(y))=\tilde{x} \tilde{y}-x \tilde{y}-\sigma(y) \tilde{x}+x \sigma(y),
$$

and we have

$$
\tilde{D}(f)=D(x y)-x D(y)-\sigma(y) D(x)+x \sigma(y) D(1)=0
$$

because $D$ is a twisted derivation (and in particular $D(1)=0$ ). It follows that $\tilde{D}$ factors through $P / I \sigma(I)$ and we may consider the induced map $u: \Omega_{\sigma}^{1} \rightarrow M$. It only remains to notice that we have

$$
\forall x \in A, \quad u(d(x))=\tilde{D}(d(x))=\tilde{D}(\tilde{x}-x)=D(x)-x D(1)=D(x) .
$$

Remark There exists a very elegant proof of this last result through the theory of bimodules. It is based on the fact (see proposition 17 in [7], Chapter III, section 10) that $I$ is universal for bimodule derivations: there exists a natural isomorphism

$$
\begin{gathered}
\operatorname{Hom}_{A-\operatorname{Bim}}(I, M) \stackrel{\simeq}{\longrightarrow} \operatorname{Der}_{R}(A, M), \\
u \longmapsto D:=u \circ d
\end{gathered}
$$

where the right-hand side stands for bimodule derivations (see proposition 1.4.2.1 of [2]).

As an immediate consequence of the proposition, if we write $T_{A / R, \sigma}:=\operatorname{Der}_{R, \sigma}(A, A)$, that we will often abbreviate to $T_{\sigma}$, we obtain the following:

Corollary 2.5. The A-module $T_{A / R, \sigma}$ is the dual of $\Omega_{A / R, \sigma}^{1}$.

Proposition 2.6. Assume that $\Omega_{A / R, \sigma}^{1}$ is projective of finite rank. Then, if $M$ is an A-module, we have the following:

1. If $R \rightarrow R^{\prime}$ is a base extension and $A^{\prime}=R^{\prime} \otimes_{R} A$, then there exists a canonical isomorphism

$$
R^{\prime} \otimes_{R} \operatorname{Der}_{R, \sigma}(A, M) \simeq \operatorname{Der}_{R^{\prime}, \sigma}\left(A^{\prime}, A^{\prime} \otimes_{A} M\right)
$$

2. If $B$ is a twisted A-algebra, there exists a canonical map

$$
B \otimes_{A} \operatorname{Der}_{R, \sigma}(A, M) \leftarrow \operatorname{Der}_{R, \sigma}\left(B, B \otimes_{A} M\right) .
$$

It is injective (resp. bijective) when $B$ is a quotient (resp. a localization) of $A$.

Proof Both assertions follow from proposition 2.3 More precisely, in the first case, we have

$$
R^{\prime} \otimes_{R} \operatorname{Der}_{R, \sigma}(A, M) \simeq R^{\prime} \otimes_{R} \operatorname{Hom}_{A}\left(\Omega_{A / R, \sigma}^{1}, M\right) \simeq \operatorname{Hom}_{A}\left(\Omega_{A / R, \sigma}^{1}, A^{\prime} \otimes_{A} M\right)
$$

because $\Omega_{A / R, \sigma}^{1}$ is projective of finite rank, and then

$$
\begin{aligned}
\operatorname{Hom}_{A}\left(\Omega_{A / R, \sigma}^{1}, A^{\prime} \otimes_{A} M\right) & \simeq \operatorname{Hom}_{A^{\prime}}\left(A^{\prime} \otimes_{A} \Omega_{A / R, \sigma}^{1}, A^{\prime} \otimes_{A} M\right) \\
& \simeq \operatorname{Hom}_{A^{\prime}}\left(\Omega_{A^{\prime} / R^{\prime}, \sigma}^{1}, A^{\prime} \otimes_{A} M\right) \simeq \operatorname{Der}_{R^{\prime}, \sigma}\left(A^{\prime}, A^{\prime} \otimes_{A} M\right) .
\end{aligned}
$$


The proof of the second assertion follows exactly the same lines with the same arguments. We have

$$
\begin{gathered}
B \otimes_{A} \operatorname{Der}_{R, \sigma}(A, M) \simeq B \otimes_{A} \operatorname{Hom}_{A}\left(\Omega_{A / R, \sigma}^{1}, M\right) \simeq \operatorname{Hom}_{A}\left(\Omega_{A / R, \sigma}^{1}, B \otimes_{A} M\right) \simeq \\
\operatorname{Hom}_{B}\left(B \otimes_{A} \Omega_{A / R, \sigma}^{1}, B \otimes_{A} M\right) \leftarrow \operatorname{Hom}_{A}\left(\Omega_{B / R, \sigma}^{1}, B \otimes_{A} M\right) \simeq \operatorname{Der}_{R, \sigma}\left(B^{\prime}, B \otimes_{A} M\right) .
\end{gathered}
$$

And the only map which is not always an isomorphism will be injective (resp. bijective) when $B$ is a quotient (resp. a localization) of $A$.

The following immediate consequence is worth stating:

Corollary 2.7. If $\Omega_{A / R, \sigma}^{1}$ is projective of finite rank, then $\mathrm{T}_{A / R, \sigma}$ and $\Omega_{A / R, \sigma}^{1}$ are dual to each other and we have

1. If $R \rightarrow R^{\prime}$ is a base extension and $A^{\prime}=R^{\prime} \otimes_{R} A$, then there exists a canonical isomorphism

$$
R^{\prime} \otimes_{R} \mathrm{~T}_{A / R, \sigma} \simeq \mathrm{T}_{A^{\prime} / R^{\prime}, \sigma^{\prime}}
$$

2. If $B$ is a twisted localization of $A$, then there exists a canonical isomorphism

$$
B \otimes{ }_{A} \mathrm{~T}_{A / R, \sigma} \simeq \mathrm{T}_{B / R, \sigma} .
$$

Definition 2.8. A twisted connection on an $A$-module $M$ is an $R$-linear map

$$
\nabla: M \mapsto M \otimes_{A} \Omega_{A / R, \sigma}^{1}
$$

such that

$$
\forall s \in M, \forall x \in A, \quad \nabla(x s)=s \otimes \mathrm{d}(x)+\sigma(x) \nabla(s) .
$$

An $A$-linear map between two $A$-modules with twisted connections is said to be horizontal if it is compatible with the connections.

Clearly, $A$-modules endowed with a connection and horizontal maps form a category $\nabla_{\sigma_{A}}-$ Mod.

Remark This definition is compatible with definition 2.2.1 by André in [2]. In particular, all the tannakian formalism applies but this is not what we are interested in.

Recall from [23] that if $D$ is a twisted derivation of $A$, then a twisted $D$-derivation of an $A$-module $M$ is an $R$-linear endomorphism $D_{M}$ of $M$ that satisfies the twisted Leibnitz rule:

$$
\forall x \in A, \forall s \in M, \quad D_{M}(x s)=D(x) s+\sigma_{A}(x) D_{M}(s) .
$$

One may then consider the notion of action by twisted derivations of $T_{A / R, \sigma}$ on $M$ : it is an $R$-linear action such that whenever $D \in T_{A / R, \sigma}$, the map $D_{M}: s \mapsto D . s$ is a $D$-derivation.

Proposition 2.9. There exists a functor from the category of A-modules endowed with a twisted connection to the category of A-modules endowed with a linear action of $T_{A / R, \sigma}$ by twisted derivations. It is an equivalence (an isomorphism) when $\Omega_{A / R, \sigma}^{1}$ is free of finite rank.

Proof If $M$ is endowed with a twisted connection $\nabla: M \mapsto M \otimes_{A} \Omega_{\sigma}^{1}$ and $D$ is a twisted derivation of $A$, we may write uniquely $D=u \circ d$ with $u: \Omega_{\sigma}^{1} \rightarrow A$ and consider the composite $\operatorname{map} D_{M}:=\left(\operatorname{Id}_{M} \otimes u\right) \circ \nabla: M \rightarrow M$. Then, we will have

$$
\begin{aligned}
D_{M}(x s) & =\left(\operatorname{Id}_{M} \otimes u\right)(\nabla(x s))=\left(\operatorname{Id}_{M} \otimes u\right)(s \otimes \mathrm{d}(x)+\sigma(x) \nabla(s)) \\
& =(\mathrm{u} \circ d)(x) s+\sigma(x)\left(\operatorname{Id}_{M} \otimes u\right)(\nabla(s))=D(x) s+\sigma(x) D_{M}(s) .
\end{aligned}
$$

Conversely, assume that $M$ is endowed with an action of $T_{\sigma}$ by twisted derivations. Let $D_{1}, \ldots, D_{n}$ be a basis of $\mathrm{T}_{\sigma}$ and $\omega_{1}, \ldots, \omega_{n}$ be the dual basis in $\Omega_{\sigma}^{1}$. Then, we can define $\nabla(s)=\sum D_{i, M}(s) \otimes \omega_{i}$ and check that

$$
\begin{aligned}
\nabla(x s) & =\sum D_{i, M}(x s) \otimes \omega_{i}=\left(\sum D_{i}(x) s+\sigma(x) D_{i, M}(s)\right) \otimes \omega_{i} \\
& =s \otimes \sum D_{i}(x) \omega_{i}+\sigma(x) \sum D_{i, M}(s) \otimes \omega_{i}=s \otimes \mathrm{d}(x)+\sigma(x) \nabla(s) .
\end{aligned}
$$

Clearly, this is an inverse to the previous functor. 
Proposition 2.10. If $x \in A$ is a twisted coordinate on $A$, then $\Omega_{A / R, \sigma}^{1}$ is free of rank 1 generated by $\mathrm{d} x$. Moreover, there exists a unique twisted derivation $\partial_{x, \sigma}$ of $A$ such that $\partial_{x, \sigma}(x)=1$ and we have

$$
\forall D \in T_{A / R, \sigma}, \quad D=D(x) \partial_{x, \sigma}
$$

Proof The first assertion is a particular case of corollary 1.13 The second one then follows from corollary 2.5 .

In particular, we see that a twisted coordinate is also a coordinate in the sense of [23]. In order to lighten the notations, we will usually drop the index $x$ but we must not forget that $\partial_{\sigma}$ depends on the choice of $x$. Also, we would rather write $\partial_{A, \sigma}$ than $\partial_{\sigma_{A}}$ when we want to make clear the dependence on $A$.

If $x$ is a twisted coordinate on $A$, one may consider the twisted Weyl algebra $\mathrm{D}_{A / R, \sigma, \partial}$ (see [23] for example), that we will usually denote by $\mathrm{D}_{A / R, \sigma}$ and sometimes simply by $\mathrm{D}_{\sigma}$. This is the non commutative polynomial ring in one variable $\partial_{\sigma}$ over $A$ with the commutation rule

$$
\forall z \in A, \quad \partial_{\sigma} z=\partial_{A, \sigma}(z)+\sigma_{A}(z) \partial_{\sigma} .
$$

Moreover, there exists an equivalence (an isomorphism) of categories $\mathrm{D}_{A / R, \sigma}-\mathrm{Mod} \simeq \partial_{A, \sigma}-\operatorname{Mod}$ where the later denotes the category of $A$-modules $M$ endowed with a $\partial_{A, \sigma}$-derivation.

Proposition 2.11. Assume that $x$ is a twisted coordinate on A. Then, there exists an equivalence (an isomorphism) of categories

$$
\nabla_{\sigma_{A}}-\operatorname{Mod} \simeq D_{A / R, \sigma}-\operatorname{Mod}
$$

Proof Follows from proposition 2.9

\section{Twisted binomial coefficient theorem for principal parts}

We prove here the main theorem that will allow us to recover twisted differential operators from principal parts. We use same notations as before.

In section 1 we introduced the canonical map (it is a morphism of $R$-algebras)

$$
\begin{aligned}
& P_{A / R} \stackrel{\delta_{A / R}}{\longrightarrow} P_{A / R} \otimes_{A} P_{A / R} . \\
& x \tilde{y} \longmapsto x \otimes \tilde{y}
\end{aligned}
$$

We want to investigate the interaction between $\sigma_{A}$ and $\delta_{A / R}$.

Recall that we also considered in section 1 the maps

$$
\begin{gathered}
P_{A / R} \stackrel{\sigma_{P}}{\longrightarrow} P_{A / R}, \\
x \tilde{y} \longmapsto \sigma_{A}(x) \tilde{y}
\end{gathered}
$$

which is an $R$-linear ring homomorphism, and

$$
\begin{aligned}
& A \stackrel{d_{A / R}}{\longrightarrow} P_{A / R}, \\
& x \longmapsto \tilde{x}-x
\end{aligned}
$$

which is only $R$-linear. As usual, we will drop the subscripts in order to lighten the notations, hoping that the meaning will always be clear from the context.

Lemma 3.1. For all $i=0, \ldots, n$, we have in $P \otimes_{A} P$ :

$$
\forall x \in A, \quad \delta\left(\sigma^{n}(d(x))\right)=1 \otimes \sigma^{i}(d(x))+\sigma^{n-i}\left(d\left(\sigma^{i}(x)\right)\right) \otimes 1 .
$$


Proof We do the computations in $A \otimes_{R} A \otimes_{R} A$. The right hand side is

$$
\begin{aligned}
& 1 \otimes\left(1 \otimes x-\sigma^{i}(x) \otimes 1\right)+\sigma^{n-i}\left(1 \otimes \sigma^{i}(x)-\sigma^{i}(x) \otimes 1\right) \otimes 1 \\
& =1 \otimes 1 \otimes x-1 \otimes \sigma^{i}(x) \otimes 1+1 \otimes \sigma^{i}(x) \otimes 1-\sigma^{n}(x) \otimes 1 \otimes 1 \\
& =1 \otimes 1 \otimes x-\sigma^{n}(x) \otimes 1 \otimes 1 .
\end{aligned}
$$

If we develop the left hand side, we obtain exactly the same thing:

$$
\delta\left(\sigma^{n}(d(x))\right)=\delta\left(1 \otimes x-\sigma^{n}(x) \otimes 1\right)=1 \otimes 1 \otimes x-\sigma^{n}(x) \otimes 1 \otimes 1 .
$$

We endow $P \otimes_{A} P$ with the endomorphism $\sigma_{P} \otimes_{A} \operatorname{Id}_{P}$ (which is the same thing as $\sigma_{A} \otimes_{R} \operatorname{Id}_{A} \otimes_{R} \operatorname{Id}_{A}$ on $\left.A \otimes_{R} A \otimes_{R} A\right)$.

Proposition 3.2. The map $\delta: P \rightarrow P \otimes_{A} P$ is a morphism of twisted $R$-algebras.

Proof This is the case $n=1$ and $i=0$ of lemma 3.1. More precisely, if $x \in A$ and $\xi=\tilde{x}-x$, we have

$$
\delta(\sigma(\xi))=1 \otimes \xi+\sigma(\xi) \otimes 1=\sigma(\delta(\xi))
$$

Proposition 3.3. We have in $P \otimes_{A} P$ :

$$
\forall n \in \mathbb{N}, \quad \delta\left(I^{(n)}\right) \subset \sum_{i=0}^{n} I^{(i)} \otimes I^{(n-i)} .
$$

Proof First of all, since $I$ is generated by the image of $d$, it follows from lemma 3.1 that for all $i=0, \ldots, n$, we have

$$
\delta\left(\sigma^{n}(I)\right) \subset P \otimes \sigma^{i}(I)+\sigma^{n-i}(I) \otimes P .
$$

Using induction, we obtain

$$
\begin{aligned}
\delta\left(I^{(n+1)}\right) & \left.=\delta\left(I^{(n)}\right) \delta\left(\sigma^{n}(I)\right)\right) \subset \sum_{i=0}^{n}\left(I^{(i)} \otimes I^{(n-i)}\right)\left(P \otimes \sigma^{n-i}(I)+\sigma^{i}(I) \otimes P\right) \\
& \subset \sum_{i=0}^{n}\left(I^{(i)} \otimes I^{(n-i+1)}\right)+\sum_{i=0}^{n}\left(I^{(i+1)} \otimes I^{(n-i)}\right) \subset \sum_{i=0}^{n+1} I^{(i)} \otimes I^{(n+1-i)} .
\end{aligned}
$$

Corollary 3.4. For all $m, n \in \mathbb{N}$, we have in $P \otimes_{A} P$ :

$$
\delta\left(I^{(n+m+1)}\right) \subset P \otimes_{A} I^{(m+1)}+I^{(n+1)} \otimes_{A} P .
$$

In other words, $\delta$ induces a map

$$
P_{(n+m)_{\sigma}} \stackrel{\delta_{n, m}}{\longrightarrow} P_{(n)_{\sigma}} \otimes_{A} P_{(m)_{\sigma}} .
$$

Proof If $0 \leq i \leq m+n+1$, we have either $i>n$ and then $I^{(i)} \subset I^{(n+1)}$ or else $i \leq n$ so that $m+n+1-i>m$ and then $I^{(m+n+1-i)} \subset I^{(m+1)}$.

Going to the limit, we obtain a canonical homomorphism of $R$-algebras

$$
\widehat{P}_{\sigma} \stackrel{\widehat{\delta}}{\longrightarrow} \widehat{P}_{\sigma} \widehat{\otimes}_{A} \widehat{P}_{\sigma}
$$

where the right hand side is, by definition, the inverse limit of all the $P_{(n)_{\sigma}} \otimes_{A} P_{(m)_{\sigma}}$. In other words, we obtain a comultiplication on $\widehat{P}_{\sigma}$ that will allow us to turn its "dual" into a ring (more on this later).

We finish this section with the quantum binomial theorem for principal parts: 
Theorem 3.5. let $A$ be a twisted commutative $R$-algebra and $x \in A$ such that $\sigma(x)=q x+h$ with $q, h \in R$. If we set $\xi=\tilde{x}-x$, then, we have

$$
\delta\left(\xi^{(n)}\right):=\sum_{i=0}^{n}\left(\begin{array}{l}
n \\
i
\end{array}\right)_{q} \xi^{(n-i)} \otimes \xi^{(i)} .
$$

Proof The formula is proved to be correct by induction on $n$. First of all, since $\delta$ is a ring homomorphism, we have

$$
\left.\delta\left(\xi^{(n+1)}\right)=\delta\left(\xi^{(n)} \sigma^{n}(\xi)\right)=\delta\left(\xi^{(n)}\right) \delta\left(\sigma^{n}(\xi)\right)\right) .
$$

Using induction and lemma 3.6 below, we get

$$
\begin{aligned}
\delta\left(\xi^{(n+1)}\right) & =\sum_{i=0}^{n}\left(\begin{array}{c}
n \\
i
\end{array}\right)_{q}\left(\xi^{(n-i)} \otimes \xi^{(i)}\right)\left(1 \otimes \sigma^{i}(\xi)+q^{i} \sigma^{n-i}(\xi) \otimes 1\right) \\
& =\sum_{i=0}^{n}\left(\begin{array}{c}
n \\
i
\end{array}\right)_{q} \xi^{(n-i)} \otimes \xi^{(i)} \sigma^{i}(\xi)+\sum_{i=0}^{n}\left(\begin{array}{c}
n \\
i
\end{array}\right)_{q} q^{i} \xi^{(n-i)} \sigma^{n-i}(\xi) \otimes \xi^{(i)} \\
& =\sum_{i=0}^{n}\left(\begin{array}{c}
n \\
i
\end{array}\right)_{q} \xi^{(n-i)} \otimes \xi^{(i+1)}+\sum_{i=0}^{n}\left(\begin{array}{c}
n \\
i
\end{array}\right)_{q} q^{i} \xi^{(n-i+1)} \otimes \xi^{(i)} \\
& =\sum_{i=1}^{n+1}\left(\begin{array}{c}
n \\
i-1
\end{array}\right)_{q} \xi^{(n-i+1)} \otimes \xi^{(i)}+\sum_{i=0}^{n}\left(\begin{array}{c}
n \\
i
\end{array}\right)_{q} q^{i} \xi^{(n-i+1)} \otimes \xi^{(i)} \\
& \left.=\sum_{i=0}^{n+1}\left(\begin{array}{c}
n \\
i-1
\end{array}\right)_{q}+q^{i\left(\begin{array}{c}
n \\
i
\end{array}\right)_{q}}\right)_{\xi^{(n+1-i)} \otimes \xi^{(i)}}^{n+1}\left(\begin{array}{c}
n+1 \\
i
\end{array}\right)_{q} \xi^{(n+1-i)} \otimes \xi^{(i)} . \\
& =\sum_{i=0}
\end{aligned}
$$

Lemma 3.6. Under the hypothesis of the proposition, we have for all $i=0, \ldots n$,

$$
\delta\left(\sigma^{n}(\xi)\right)=1 \otimes \sigma^{i}(\xi)+q^{i} \sigma^{n-i}(\xi) \otimes 1 .
$$

Proof We have

$$
d(\sigma(x))=\widetilde{\sigma(x)}-\sigma(x)=\widetilde{q x+h}-(q x+h)=q(\tilde{x}-x)=q \xi .
$$

The analogous result holds for the endomorphism $\sigma^{i}$. It follows that $d\left(\sigma^{i}(x)\right)=q^{i} \xi$ and we finish with lemma 3.1

\section{Twisted differential operators of infinite level}

We are now able to define the ring of twisted differential operators (of infinite level). We keep the previous notations.

Definition 4.1. If $M$ and $N$ are two A-modules, then a twisted differential operator $\varphi: M \rightarrow N$ of order at most $n$ (and infinite level) is an $R$-linear map whose $A$-linearization

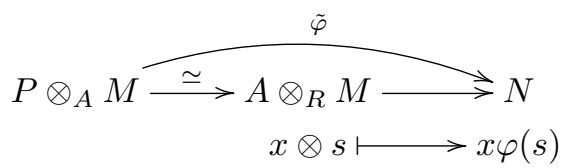

factors through $P_{(n)_{\sigma}} \otimes_{A} M$. 
Note that the condition means that the restriction of $\tilde{\varphi}$ to $I^{(n+1)} \otimes_{A} M$ is zero. We might still write $\tilde{\varphi}$ for the map induced on $P_{(n)_{\sigma}} \otimes_{A} M$ when there is no risk of confusion.

We denote by $\operatorname{Diff}_{n, \sigma}(M, N)$ the set of all twisted differential operators of order at most $n$. Thus, we have a canonical isomorphism

$$
\operatorname{Diff}_{n, \sigma}(M, N) \simeq \operatorname{Hom}_{A}\left(P_{(n)_{\sigma}} \otimes_{A} M, N\right) .
$$

where $P_{(n)_{\sigma}}$ is seen as an $A$-module for the action on the right with respect to $\otimes_{A}$ and for the action on the left with respect to $\operatorname{Hom}_{A}$. In particular, Diff $n, \sigma(M, N)$ has a natural structure of $P_{(n)_{\sigma}}$-module given by $x \tilde{y} \cdot \varphi:=x \circ \varphi \circ y$, where multiplication by $y$ takes place in $M$ while we multiply by $x$ in $N$.

We will also denote by $\operatorname{Diff}_{\sigma}(M, N)$ the set of all twisted differential operators of any order so that

$$
\operatorname{Diff}_{\sigma}(M, N) \simeq \lim _{\longrightarrow} \operatorname{Hom}_{A}\left(P_{(n)_{\sigma}} \otimes_{A} M, N\right) .
$$

In particular, we see that Diff $\sigma(M, N)$ has a natural structure of $\widehat{P}_{\sigma}$-module.

In the case $N=M$, we will write $\operatorname{Diff}_{n, \sigma}(M)$ and $\operatorname{Diff}_{\sigma}(M)$. One also sets $\mathrm{D}_{A / R, \sigma}^{(\infty)}:=\operatorname{Diff}_{\sigma}(A)$ and we will often drop the index $A / R$ and simply write $\mathrm{D}_{\sigma}^{(\infty)}$.

Definition 4.2. Let $x$ be a twisted coordinate on $A$ and $\xi=\tilde{x}-x$. Then, the standard basis of $\operatorname{Diff}_{n, \sigma}(A)$ is the basis $\partial_{\sigma}^{[k]}$ dual to the images of the $\xi^{(k)}$ in $P_{(n)_{\sigma}}$. We call $\partial_{\sigma}^{[k]}$ the standard twisted divided differential operator of order $k$ associated to $x$.

In other words (we will come back to this in section 7), the canonical basis is characterized by the property

$$
\forall k, n \in \mathbb{N}, \quad \widetilde{\partial_{\sigma}^{[k]}}\left(\xi^{(n)}\right)= \begin{cases}1 & \text { if } k=n \\ 0 & \text { otherwise }\end{cases}
$$

where $\widetilde{\partial_{\sigma}^{[k]}}$ denotes the $A$-linearization of the $R$-linear endomorphism $\partial_{\sigma}^{[k]}$ of $A$.

Thus, when $x$ is a twisted coordinate, any $\varphi \in D_{\sigma}^{(\infty)}$ can be uniquely written as a finite sum $\sum z_{k} \partial_{\sigma}^{[k]}$ with $z_{k} \in A$ (and conversely, any such sum is in $D_{\sigma}^{(\infty)}$ )

The next proposition shows that the $A$-module of twisted differential operators could have been also defined by induction on the order $n$ (this is sometimes more convenient and does not require to work out the theory of principal parts). Instead, it uses the notion of twisted bracket

$$
\forall \varphi \in \operatorname{Hom}_{R}(M, N), \forall x \in A, \quad[\varphi, x]_{\sigma}=\varphi \circ x-\sigma(x) \circ \varphi
$$

already used in [23]. We will need this intermediate result:

Lemma 4.3. Let $M, N$ be two A-modules, $\varphi \in \operatorname{Hom}_{R}(M, N)$ and $x \in A$. If we set $\varphi_{x}:=[\varphi, x]_{\sigma}$, then,

$$
\tilde{\varphi}_{x}=\tilde{\varphi} \circ\left(\sigma(\xi) \otimes_{A} \operatorname{Id}_{M}\right): P \otimes_{A} M \rightarrow N
$$

Proof We do the computations in $A \otimes_{R} M$. Let $y \in A$ and $s \in M$, we have

$$
\begin{aligned}
\tilde{\varphi}(\sigma(\xi)(y \otimes s)) & =\tilde{\varphi}((1 \otimes x-\sigma(x) \otimes 1)(y \otimes s))=\tilde{\varphi}(y \otimes x s)-\tilde{\varphi}(\sigma(x) y \otimes s) \\
& =y \varphi(x s)-\sigma(x) y \varphi(s)=y \varphi_{x}(s)=\tilde{\varphi}_{x}(y \otimes s) . \quad \square
\end{aligned}
$$

Proposition 4.4. Let $M, N$ be two A-modules and $\varphi \in \operatorname{Hom}_{R}(M, N)$. Then, for all $n \in \mathbb{N}$, we have

$$
\varphi \in \operatorname{Diff}_{n, \sigma}(M, N) \Leftrightarrow \forall x \in A,[\varphi, x]_{\sigma^{n}} \in \operatorname{Diff}_{n-1, \sigma}(M, N) .
$$

Note that we can start the induction process with $\operatorname{Diff}_{0, \sigma}(M, N)=\operatorname{Hom}_{A}(M, N)$ or with $\operatorname{Diff}_{-1, \sigma}(M, N)=0$ if we prefer. 
Proof For $x \in A$, we set $\varphi_{x}:=[\varphi, x]_{\sigma^{n}}$. Then, we consider the linearizations

$$
\tilde{\varphi}, \tilde{\varphi}_{x}: P \otimes_{A} M \rightarrow N
$$

of $\varphi$ and $\varphi_{x}$, respectively, and apply lemma 4.3 to $\sigma^{n}$ so that

$$
\tilde{\varphi}_{x}=\tilde{\varphi} \circ\left(\sigma^{n}(\xi) \otimes_{A} \operatorname{Id}_{M}\right) .
$$

Thus we see that $\tilde{\varphi}=0$ on $I^{(n+1)} \otimes_{A} M=I^{(n)} \sigma^{n}(I) \otimes_{A} M$ if and only if $\tilde{\varphi}_{x}=0$ on $I^{(n)} \otimes_{A} M$ for all $x \in A$. In other words, $\tilde{\varphi}$ factors through $P_{(n)_{\sigma}} \otimes_{A} M$ if and only if all $\tilde{\varphi}_{x}$ factor through $P_{(n-1)_{\sigma}} \otimes_{A} M$.

Corollary 4.5. A twisted differential operator of order at most $n$ (and infinite level) from $M$ to $N$ is an R-linear map $\varphi: M \rightarrow N$ such that

$$
\forall x_{0}, \ldots, x_{n} \in A, \quad\left[\left[\cdots\left[\left[\varphi, x_{n}\right]_{\sigma^{n}}, x_{n-1}\right]_{\sigma^{n-1}} \cdots\right]_{\sigma}, x_{0}\right]=0 .
$$

Remarks 1. Be careful that, with the notations of Valery Lunts and Alexander L. Rosenberg in 24], our $\operatorname{Diff}_{\sigma}(M, N)$ is different from their $\operatorname{Diff}\left(M, N^{\sigma}\right)$ which is defined by the condition

$$
\forall x_{0}, \ldots, x_{n} \in A, \quad\left[\left[\cdots\left[\left[\varphi, x_{n}\right]_{\sigma}, x_{n-1}\right]_{\sigma} \cdots\right]_{\sigma}, x_{0}\right]=0 .
$$

They only coincide when $n=0,1$.

2. Our Diff ${ }_{\sigma}(M, N)$ should however coincide with some flavor of the $\operatorname{Diff}_{\beta}(M, N)$ of Lunts and Rosenberg. More precisely, in order to define this module, they need a $G$-grading on $A$ and a bilinear map $\beta: G \times G \rightarrow R^{\times}$(they use $k$ and $R$ for our $R$ and $A$ ). In the simplest non trivial case $A=R[x]$ and $\beta(m, n)=q^{-m n}$, we believe that their $D_{\beta}(A)$ coincides with our $D_{A / R, \sigma}^{(\infty)}$ but their $D_{q}(A)$ is bigger (see [20] for example).

3. Charlotte Hardouin introduces in [19, definition 2.4, what she calls an iterative q-difference ring or $\mathrm{ID}_{q}$-ring for short. She chooses some non zero $q \in K$ where $K$ is a fixed algebraically closed field and endows the field $A:=K(x)$ of rational functions on $K$ with the automorphism $\sigma(x)=q x$. Then, if we look carefully at her conditions, we see that an $\mathrm{ID}_{q}$-ring is a finitely generated $A$-algebra $B$ with a structure of $D_{A / K, \sigma}^{(\infty)}$-module, denoted by $(\varphi, y) \mapsto \varphi(y)$, such that the map $y \mapsto \sigma(y)$ is an automorphism of the $\operatorname{ring} B$ and

$$
\forall k \in \mathbb{N}, \forall y, z \in B, \quad \partial_{\sigma}^{[k]}(y z)=\sum_{i+j=k}\left(\sigma^{i} \partial_{\sigma}^{[j]}\right)(y) \partial_{\sigma}^{[i]}(z)
$$

Note that $B$ becomes a twisted $A$-algebra, that is in fact inversive (which simply means that $\sigma$ is bijective on $B$, a condition that is built into Hardouin's definition), and that condition (4.1) is automatic if the $q$-characteristic of $B$ is zero.

Proposition 4.6. Composition of twisted differential operators gives a twisted differential operator. Moreover, its order is at most the sum of the order of the components.

Proof We let $\varphi: M \rightarrow N$ be a twisted differential operator of order $n$ and $\psi: L \rightarrow M$ a twisted differential operator of order $m$ and consider the factorization

$$
P \otimes_{A} L \stackrel{\delta \otimes \operatorname{Id}}{\longrightarrow} P \otimes_{A} P \otimes_{A} L \stackrel{\operatorname{Id} \otimes_{A} \tilde{\psi}}{\longrightarrow} P \otimes_{A} M \stackrel{\tilde{\varphi}}{\longrightarrow} N
$$

of lemma 1.2 The map $\tilde{\varphi}$ factors through $P_{(n)_{\sigma}} \otimes_{A} M$ and $\operatorname{Id} \otimes \tilde{\psi}$ factors through $P \otimes_{A} P_{(m)_{\sigma}} \otimes_{A} L$. Therefore, their composite factors through $P_{(n)_{\sigma}} \otimes_{A} P_{(m)_{\sigma}} \otimes_{A} L$ and it follows from corollary 3.4 that the whole thing will factor through $P_{(n+m)_{\sigma}} \otimes L$. Thus, $\varphi \circ \psi$ is a twisted differential operator of order at most $m+n$. 
It is sometimes useful to have a general formula for the commutation of twisted differential operators with the twisted coordinate $x$ :

Proposition 4.7. We have

$$
\forall k \in \mathbb{N} \backslash\{0\}, \quad \partial_{\sigma}^{[k]} \circ x=\sigma^{k}(x) \partial_{\sigma}^{[k]}+\partial_{\sigma}^{[k-1]} .
$$

Proof In order to make the proof easier to understand, we will still write $\tilde{x}=1 \otimes x \in P$ but we will denote the multiplication maps as

$$
\begin{array}{lll}
A \stackrel{m_{x}}{\longrightarrow} A & \text { and } \quad P \stackrel{m_{\tilde{x}}}{\longrightarrow} P \\
y \longmapsto &
\end{array}
$$

Then, it is easy to see that $m_{\tilde{x}}$ splits as

$$
P \stackrel{\delta}{\longrightarrow} P \otimes_{A} P \stackrel{\operatorname{Id}_{P} \otimes_{A} \widetilde{m_{x}}}{\longrightarrow} P
$$

where $\widetilde{m_{x}}$ denotes the linearization of $m_{x}$. It then follows from lemma 1.2 that

$$
\widetilde{\partial_{\sigma}^{[k]} \circ m_{x}}=\widetilde{\partial_{\sigma}^{[k]}} \circ m_{\tilde{x}} .
$$

On the other hand, we have

$$
\xi^{(n+1)}=\xi^{(n)} \sigma^{n}(\xi)=\xi^{(n)}\left(\tilde{x}-\sigma^{n}(x)\right)=\tilde{x} \xi^{(n)}-\sigma^{n}(x) \xi^{(n)},
$$

from which we derive

$$
\tilde{x} \xi^{(n)}=\xi^{(n+1)}+\sigma^{n}(x) \xi^{(n)} .
$$

Putting all these together, we obtain

$$
\begin{aligned}
& \left(\widetilde{\partial_{\sigma}^{[k]} \circ m_{x}}\right)\left(\xi^{(n)}\right)=\left(\widetilde{\partial_{\sigma}^{[k]}} \circ m_{\tilde{x}}\right)\left(\xi^{(n)}\right) \\
& =\widetilde{\partial_{\sigma}^{[k]}}\left(\tilde{x} \xi^{(n)}\right) \\
& =\widetilde{\partial_{\sigma}^{[k]}}\left(\xi^{(n+1)}+\sigma^{n}(x) \xi^{(n)}\right) \\
& =\left\{\begin{array}{l}
1 \quad \text { if } \quad n=k-1 \\
\sigma^{k}(x) \text { if } n=k \\
0 \text { otherwise }
\end{array} .\right.
\end{aligned}
$$

In other words, we have

$$
\partial_{\sigma}^{[k]} \circ m_{x}=\sigma^{k}(x) \partial_{\sigma}^{[k]}+\partial_{\sigma}^{[k-1]} .
$$

Corollary 4.8. If $x \in A^{\times}$, then

$$
\forall k \in \mathbb{N} \backslash\{0\}, \quad \partial_{\sigma}^{[k]} \circ x^{-1}=\sum_{i=0}^{k} \frac{(-1)^{i}}{\prod_{j=0}^{i} \sigma^{k-j}(x)} \partial_{\sigma}^{[k-i]} .
$$

Proof Using for a moment the convention $\partial_{\sigma}^{[-1]}=0$, which makes proposition 4.7 formally valid for $k=0$, we compute

$$
\begin{aligned}
\sum_{i=0}^{k} \frac{(-1)^{i}}{\prod_{j=0}^{i} \sigma^{k-j}(x)} \partial_{\sigma}^{[k-i]} \circ x & =\sum_{i=0}^{k} \frac{(-1)^{i}}{\prod_{j=0}^{i} \sigma^{k-j}(x)}\left(\sigma^{k-i}(x) \partial_{\sigma}^{[k-i]}+\partial_{\sigma}^{[k-i-1]}\right) \\
& =\sum_{i=0}^{k} \frac{(-1)^{i}}{\prod_{j=0}^{i-1} \sigma^{k-j}(x)} \partial_{\sigma}^{[k-i]}+\sum_{i=0}^{k-1} \frac{(-1)^{i}}{\prod_{j=0}^{i} \sigma^{k-j}(x)} \partial_{\sigma}^{[k-i-1]}=\partial_{\sigma}^{[k]}
\end{aligned}
$$


Proposition 4.9. Let $M, N$ be two A-modules.

1. Let $R \rightarrow R^{\prime}$ be any morphism of commutative rings and $A^{\prime}:=R^{\prime} \otimes_{R} A$, endowed with $\operatorname{Id}_{R^{\prime}} \otimes_{R} \sigma_{A}$. Then, we have

$$
\operatorname{Diff}_{\sigma_{A^{\prime}}}\left(A^{\prime} \otimes_{A} M, A^{\prime} \otimes_{A} N\right) \simeq A^{\prime} \otimes_{A} \operatorname{Diff}_{\sigma_{A}}(M, N) .
$$

2. If $A \rightarrow B$ is a twisted localization and $M$ is finitely presented, then we have

$$
\operatorname{Diff}_{\sigma_{B}}\left(B \otimes_{A} M, B \otimes_{A} N\right) \simeq B \otimes_{A} \operatorname{Diff}_{\sigma_{A}}(M, N) .
$$

Proof Follows from propositions 1.6 and 1.7 and the fact that direct limits commute with tensor product (and localization is flat).

Remark 1. As a particular case, we will have

$$
\mathrm{D}_{A^{\prime} / R^{\prime}, \sigma^{\prime}}^{(\infty)} \simeq A^{\prime} \otimes_{A} \mathrm{D}_{A / R, \sigma}^{(\infty)} \quad \text { and } \quad \mathrm{D}_{B / R, \sigma}^{(\infty)} \simeq B \otimes_{A} \mathrm{D}_{A / R, \sigma}^{(\infty)} .
$$

2. When $A$ is a quantum $R$-algebra, we will always have (see the remark following proposition 1.10 a natural isomorphism of $A$-modules

$$
\mathrm{D}_{A / R, \sigma}^{(\infty)} \simeq A \otimes_{R[x]} \mathrm{D}_{R[x] / R, \sigma}^{(\infty)} .
$$

Note however that the ring structure (or equivalently the action on $A$ ) plays a fundamental role.

Proposition 4.10. We have $\operatorname{Diff}_{0, \sigma}(A)=A$ and $\operatorname{Diff}_{1, \sigma}(A)=A \oplus \mathrm{T}_{A / R, \sigma}$.

Proof The first assertion follows from the fact that $P_{(0)_{\sigma}}=A$ and the second one from proposition 2.2

Recall that we introduced in [23] the ring $\overline{\mathrm{D}}_{A / R, \sigma}$ of small twisted differential operators of $A / R$ as the smallest subring of $\operatorname{End}_{R}(A)$ containing both $A$ and $\mathrm{T}_{A / R, \sigma}$. Again, we will simply write $\overline{\mathrm{D}}_{\sigma}$ when we believe that there is no risk of confusion. Then, we have the following:

Corollary 4.11. The ring of small twisted differential operators is contained inside the ring of twisted differential operators of infinite level: we have

$$
\overline{\mathrm{D}}_{A / R, \sigma} \subset \mathrm{D}_{A / R, \sigma}^{(\infty)} .
$$

When there exists a twisted coordinate, we can make the twisted Weyl algebra enter the picture and we have:

Corollary 4.12. If $x$ is a twisted coordinate on A, there exists an epi-mono factorization

$$
\mathrm{D}_{A / R, \sigma} \longrightarrow \overline{\mathrm{D}}_{A / R, \sigma} \longrightarrow \mathrm{D}_{A / R, \sigma}^{(\infty)} .
$$

Proof There exists a natural map $\mathrm{D}_{\sigma} \rightarrow \overline{\mathrm{D}}_{\sigma}$ that sends the parameter $\partial_{\sigma}$ of $\mathrm{D}_{\sigma, \partial}$ to the corresponding endomorphism $\partial_{A, \sigma}$ of $A$. And it is surjective since $\partial_{A, \sigma}$ is a generator of $\mathrm{T}_{\sigma}$.

None of the maps are bijective, even in characteristic zero. However, as we will see in theorem 6.3 below, there are some important cases where both maps are bijective.

At some point, we will need to be able to compare twisted differential operators with respect to $\sigma$ and twisted differential operators with respect to the powers (or roots) of $\sigma$. 
Proposition 4.13. For all $m>0$, if $M, N$ are two $A$-modules, we have

$$
\operatorname{Diff}_{\sigma^{m}}(M, N) \subset \operatorname{Diff}_{\sigma}(M, N) .
$$

Proof Since

$$
I^{(m n)_{\sigma}}=I \sigma(I) \cdots \sigma^{m n}(I) \subset I \sigma^{m}(I) \cdots \sigma^{m n}(I)=I^{(n)_{\sigma} m},
$$

there exists an natural surjective map

$$
P_{(n m)_{\sigma}} \rightarrow P_{(n)_{\sigma} m}
$$

from which we derive an inclusion $\operatorname{Diff}_{n, \sigma^{m}}(M, N) \subset \operatorname{Diff}_{m n, \sigma}(M, N)$.

Recall from 22 that a system of roots of $\sigma$ is a family $\underline{\sigma}:=\left\{\sigma_{n}\right\}_{n \in S}$, with $S \subset \mathbb{N}$, of $R$-linear ring endomorphisms of $A$ such that $\sigma_{n}^{m}=\sigma_{n^{\prime}}^{m^{\prime}}$ whenever $m / n=m^{\prime} / n^{\prime}$ and $\sigma_{n}^{n}=\sigma$. We will always assume that $S$ is filtering for division in the sense that if $m, m^{\prime} \in S$, then there always exists $m^{\prime \prime} \in S$ such that $m \mid m^{\prime \prime}$ and $m^{\prime} \mid m^{\prime \prime}$. We will call the pair $(A, \underline{\sigma})$ an $S$-twisted $R$-algebra.

Definition 4.14. Let $\underline{\sigma}:=\left\{\sigma_{n}\right\}_{n \in S}$ be a system of roots of $\sigma$. Then the ring of twisted differential operators (of infinite level) $\mathrm{D}_{A / R, \sigma}^{(\infty)}$ is the $R$-subalgebra of $\operatorname{End}_{R}(A)$ generated by all $\sigma_{n}$-differential operators (of infinite level) for all $n \in S$.

Proposition 4.13 then has the following consequence:

Corollary 4.15. If $\underline{\sigma}$ is a system of roots of $\sigma$, we have

$$
\mathrm{D}_{A / R, \underline{\sigma}}^{(\infty)}=\cup \mathrm{D}_{A / R, \sigma_{n}}^{(\infty)} .
$$

Note that in section 3 of 23 ] we defined exactly in the same way the ring of small twisted differential operators $\overline{\mathrm{D}}_{A / R, \sigma}$ for any family $\underline{\sigma}$ (not necessarily a root system), but we showed that the analogous statement is not true in general.

\section{$5 \quad$ Twisted taylor series}

We will develop here the formalism of twisted Taylor maps which describes the formal solutions of twisted differential modules. Notations are as before.

Lemma 5.1. If $M$ is a $\mathrm{D}_{A / R, \sigma}^{(\infty)}$-module, then the canonical map $\mathrm{D}_{A / R, \sigma}^{(\infty)} \rightarrow \operatorname{End}_{R}(M)$ induces, for all $n \in \mathbb{N}$, a $P_{A / R,(n)_{\sigma}}$-linear map

$$
\operatorname{Diff}_{n, \sigma}(A) \rightarrow \operatorname{Diff}_{n, \sigma}(M) .
$$

Hence, there exists a canonical $\widehat{P}_{\sigma}$-linear map

$$
D_{A / R}^{(\infty)} \rightarrow \operatorname{Diff}_{\sigma}(M)
$$

Proof Since the canonical map $\lambda: D_{\sigma}^{(\infty)} \rightarrow \operatorname{End}_{R}(M)$ is a morphism of $A$-algebras, it will commute with the action of $P$. More precisely, for all $x, y \in A$ and $\varphi \in D_{\sigma}^{(\infty)}$, we have (see section 4 for the definition of the action of $P$ on $D_{\sigma}^{(\infty)}$ )

$$
\lambda(x \tilde{y} \cdot \varphi)=\lambda(x \circ \varphi \circ y)=x \circ \lambda(\varphi) \circ y=x \tilde{y} \cdot \lambda(\varphi) .
$$

In particular, if $\tilde{\varphi}$ is zero on $I^{(n+1)}$, then $\widetilde{\lambda(\varphi)}$ will be zero on $I^{(n+1)} \otimes_{A} M$. It means that the image of Diff ${ }_{n, \sigma}(A)$ falls inside $\operatorname{Diff}_{n, \sigma}(M)$. 
We will usually denote by $\varphi_{M} \in \operatorname{Diff}_{\sigma}(M)$ the image of $\varphi \in D_{\sigma}^{(\infty)}$. In other words, for $\varphi \in D_{\sigma}^{(\infty)}$ and $s \in M$, we will have $\varphi_{M}(s)=\varphi s$.

Definition 5.2. A twisted Taylor structure (of infinite level) on an $A$-module $M$ is a compatible family of $A$-linear maps $\theta_{n}: M \rightarrow M \otimes_{A} P_{(n)_{\sigma}}$ (called twisted Taylor maps) with $\theta_{0}=$ Id, making commutative all the diagrams

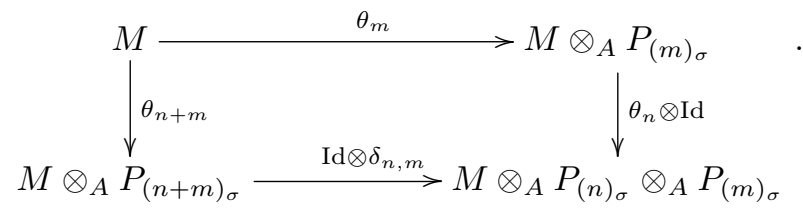

For example, the canonical twisted Taylor structure on $A$ is defined by the family of composite maps

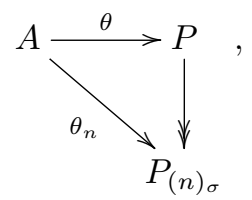

where the upper map is the Taylor map $x \mapsto \tilde{x}$ given by the action on the right (see section 1).

There exists an obvious notion of morphism of $A$-modules endowed with a twisted Taylor structure and they form a category.

Proposition 5.3. Let $M$ be an A-module endowed with a twisted Taylor structure $\left(\theta_{n}\right)_{n \in \mathbb{N}}$. Then, there exists a unique structure of $\mathrm{D}_{A / R, \sigma}^{(\infty)}$-module on $M$ such that, for all $n \in \mathbb{N}$, we have

$$
\theta_{n}(s)=\sum s_{k} \otimes f_{k} \Rightarrow \forall \varphi \in \operatorname{Diff}_{n, \sigma}(A), \varphi_{M}(s)=\sum \tilde{\varphi}\left(f_{k}\right) s_{k} .
$$

This is functorial in $M$ in the sense that any morphism of A-modules $M \rightarrow N$ which is compatible with some twisted Taylor structures on $M$ and $N$ will automatically be $\mathrm{D}_{A / R, \sigma}^{(\infty)}$-linear. Moreover, this is an equivalence (an isomorphism) of categories if all $P_{(n)_{\sigma}}$ are finite projective (for the left A-module structure).

Note that the last condition is satisfied if there exists a twisted coordinate on $A$.

Proof First of all, there exists for all $n \in \mathbb{N}$, a canonical morphism of $A$-modules

$$
M \otimes_{A} P_{(n)_{\sigma}} \rightarrow \operatorname{Hom}_{A}\left(\operatorname{Hom}_{A}\left(P_{(n)_{\sigma}}, A\right), M\right) .
$$

which is automatically $P_{(n)_{\sigma}}$-linear. Now, $A$-linear maps

$$
\theta_{n}: M \rightarrow M \otimes_{A} P_{(n)_{\sigma}}
$$

correspond bijectively to $P_{(n)_{\sigma}}$-linear maps

$$
\epsilon_{n}: P_{(n)_{\sigma}} \otimes_{A} M \rightarrow M \otimes_{A} P_{(n)_{\sigma}},
$$

and we can compose with the map $(5.2)$ in order to get

$$
P_{(n)_{\sigma}} \otimes_{A} M \rightarrow \operatorname{Hom}_{A}\left(\operatorname{Hom}_{A}\left(P_{(n)_{\sigma}}, A\right), M\right),
$$

or equivalently,

$$
\operatorname{Hom}_{A}\left(P_{(n)_{\sigma}}, A\right) \rightarrow \operatorname{Hom}_{A}\left(P_{(n)_{\sigma}} \otimes_{A} M, M\right) .
$$

In other words, we obtain $P_{(n)_{\sigma}}$-linear maps

$$
\begin{gathered}
\operatorname{Diff}_{n, \sigma}(A) \longrightarrow \operatorname{Diff}_{n, \sigma}(M) . \\
\varphi \longmapsto \varphi_{M}
\end{gathered}
$$


Formula (5.1) follows directly from the construction. Compatibility for various $n$ in (5.4) follows from compatibility for various $n$ in (5.3). We need to show that the corresponding map $\mathrm{D}_{\sigma}^{(\infty)} \rightarrow \operatorname{End}(M)$ is a morphism of rings. To do that, one can use the description of composition of twisted differential operators given in proposition 4.6 , we need to verify that the maps $(5.3)$ are compatible with $\delta$ which is exactly the condition in the definition of Taylor structure.

This construction is clearly functorial. Moreover, if $P_{(n)_{\sigma}}$ is finite projective (for the left $A$-module structure), then the map (5.2) is actually an isomorphism. And it follows from lemma 5.1 that a $\mathrm{D}_{\sigma}^{(\infty)}$-module structure on $M$ will provide us with a compatible family of maps as in (5.4).

Definition 5.4. Let $M$ be an $A$-module endowed with a twisted Taylor structure. Then, the twisted Taylor map of $M$ is the map

$$
\widehat{\theta}=\lim _{\longleftarrow} \theta_{n}: M \mapsto M \widehat{\otimes}_{A} \widehat{P}_{\sigma}:=\lim _{\longleftarrow} M \otimes_{A} P_{(n)_{\sigma}} .
$$

The twisted Taylor series of $s \in M$ is $\widehat{\theta}(s) \in M \widehat{\otimes}_{A} \widehat{P}_{\sigma}$.

There exists a commutative diagram

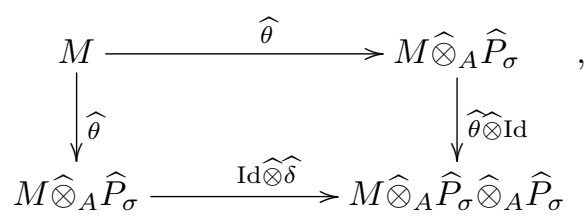

and the action of $A$ on $\widehat{P}_{\sigma}$ on the right is given by the Taylor map of $A$.

In practice, we will only consider the case of finitely presented $A$-modules $M$, and then, the completed tensor product is the usual tensor product $M \otimes_{A} \widehat{P}_{\sigma}$

We just showed in proposition 5.3 that any $\mathrm{D}_{\sigma}^{(\infty)}$-module comes with a canonical twisted Taylor structure. When there exists a twisted coordinate on $A$, we can can describe it explicitly as follows:

Proposition 5.5 (Twisted Taylor formula). Assume that $x$ is a twisted coordinate on $A$ and let $\xi=\tilde{x}-x$. If $M$ is a $\mathrm{D}_{\sigma}^{(\infty)}$-module, we have for all $n \in \mathbb{N}$,

$$
\theta_{n}(s)=\sum_{k=0}^{n} \partial_{\sigma}^{[k]}(s) \otimes \xi^{(k)} \in M \otimes_{A} P_{(n)_{\sigma}}
$$

and

$$
\widehat{\theta}(s)=\sum_{k=0}^{\infty} \partial_{\sigma}^{[k]}(s) \otimes \xi^{(k)} \in M \widehat{\otimes}_{A} \widehat{P}_{\sigma} .
$$

Proof This follows from equation $(5.1)$ : if we write

$$
\theta_{n}(s)=\sum_{k=0}^{n} s_{k} \otimes \xi^{(k)},
$$

we will have for all $l \in \mathbb{N}$,

$$
\partial_{\sigma}^{[l]}(s)=\sum_{k=0}^{n} \tilde{\partial}_{\sigma}^{[l]}\left(\xi^{(k)}\right) s_{k}=s_{l}
$$

In particular, we see that, if $z \in A$, then the image in $\widehat{P}_{\sigma}$ of $\tilde{z}=1 \otimes z \in P$ is the twisted Taylor series

$$
\widehat{\theta}(z)=\sum_{k} \partial_{\sigma}^{[k]}(z) \xi^{(k)} .
$$

This explains why it is legitimate to call the map $z \mapsto \tilde{z}$ the Taylor map. 
Examples 1. If $x$ is a twisted coordinate on $A$, we have $\widehat{\theta}(x)=x+\xi$ and

$$
\widehat{\theta}\left(x^{2}\right)=x^{2}+(x+\sigma(x)) \xi+\xi^{2} .
$$

2. Assume that $x \in A^{\times}$is an invertible twisted coordinate on $A$ and that $\sigma(x)=q x$ with $q \in R^{\times}$, then we have

$$
\widehat{\theta}\left(\frac{1}{x}\right)=\sum_{k=0}^{\infty}(-1)^{k} \frac{\xi^{(k)}}{q^{\frac{k(k+1)}{2}} x^{k+1}}=\frac{1}{x}-\frac{\xi}{q x^{2}}+\frac{\xi^{(2)}}{q^{3} x^{3}}-\cdots .
$$

Remark If $A$ is a twisted localization of $R[x]$, then there exists at most one $R$-algebra homomorphism $\widehat{\theta}: A \rightarrow \widehat{P}_{\sigma}$ such that $\widehat{\theta}(x)=\tilde{x}$. It means that the twisted Taylor map

$$
\begin{aligned}
& A \stackrel{\widehat{\theta}}{\longrightarrow} \widehat{P}_{\sigma} \\
& z \longmapsto \sum_{k=0}^{\infty} \partial_{\sigma}^{[k]}(z) \xi^{(k)}
\end{aligned}
$$

is the unique such map.

\section{Quantum differential operators}

In the quantum situation, we can be a lot more explicit as we shall see now. Thus, we assume in this section that $A$ is a quantum $R$-algebra: we are given a twisted coordinate $x$ such that $\sigma(x)=q x+h$ with $q, h \in R$.

Proposition 6.1. We have

$$
\forall k, l \in \mathbb{N}, \quad \partial_{\sigma}^{[k]} \circ \partial_{\sigma}^{[l]}=\left(\begin{array}{c}
k+l \\
l
\end{array}\right)_{q} \partial_{\sigma}^{[k+l]} .
$$

Proof It follows from lemma 1.2 that

$$
\widetilde{\partial_{\sigma}^{[k]} \circ \partial_{\sigma}^{[l]}}=\widetilde{\partial_{\sigma}^{[k]}} \circ\left(\operatorname{Id} \otimes \widetilde{\partial_{\sigma}^{[l]}}\right) \circ \delta .
$$

Thus, using theorem 3.5 , we see that

$$
\begin{aligned}
& \left(\widetilde{\partial_{\sigma}^{[k]} \circ \partial_{\sigma}^{[l]}}\right)\left(\xi^{(n)}\right)=\widetilde{\partial_{\sigma}^{[k]}}\left(\left(\operatorname{Id} \otimes \widetilde{\partial_{\sigma}^{[l]}}\right)\left(\sum_{i=0}^{n}\left(\begin{array}{c}
n \\
i
\end{array}\right)_{q} \xi^{(n-i)} \otimes \xi^{(i)}\right)\right) \\
& =\sum_{i=0}^{n}\left(\begin{array}{l}
n \\
i
\end{array}\right) \widetilde{\partial_{\sigma}^{[k]}}\left(\xi^{(n-i)} \otimes \widetilde{\partial_{\sigma}^{[l]}}\left(\xi^{(i)}\right)\right) \\
& =\left(\begin{array}{l}
n \\
l
\end{array}\right)_{q} \widetilde{\partial_{\sigma}^{[k]}}\left(\xi^{(n-l)}\right)=\left\{\begin{array}{cl}
\left(\begin{array}{c}
k+l \\
l
\end{array}\right)_{q} & \text { if } n=k+l \\
0 & \text { otherwise }
\end{array}\right.
\end{aligned}
$$

Recall from 22 that if $m \in \mathbb{N}$, we write $(m)_{q}:=\left(\begin{array}{c}m \\ 1\end{array}\right)_{q}=1+\cdots+q^{m-1}$ and we define by induction $(m)_{q} !:=(m)_{q}(m-1)_{q} !$

Corollary 6.2. We have

$$
\forall k \in \mathbb{N}, \forall z \in A, \quad \partial_{\sigma}^{k}(z)=(k)_{q} ! \partial_{\sigma}^{[k]}(z)
$$

Proof We proceed by induction on $k$ and obtain

$$
\begin{gathered}
\partial_{\sigma}^{k+1}(z)=\partial_{\sigma}^{k}\left(\partial_{\sigma}(z)\right)=(k)_{q} ! \partial_{\sigma}^{[k]}\left(\partial_{\sigma}(z)\right)=(k)_{q} !\left(\partial_{\sigma}^{[k]} \partial_{\sigma}\right)(z) \\
=(k)_{q} !\left(\begin{array}{c}
k+1 \\
1
\end{array}\right)_{q} \partial_{\sigma}^{[k+1]}(z)=(k+1)_{q} ! \partial_{\sigma}^{[k+1]}(z) . \quad \square
\end{gathered}
$$


The next result is important because it describes explicitly the relations between the different rings of twisted differential operators introduced so far. Recall from proposition 4.12 that there exists an epi-mono factorization

$$
\mathrm{D}_{A / R, \sigma} \longrightarrow \overline{\mathrm{D}}_{A / R, \sigma} \longrightarrow \mathrm{D}_{A / R, \sigma}^{(\infty)}
$$

Recall from 22 that the ring $R$ is said to be $q$-flat (resp. $q$-divisible) if $(m)_{q}$ is never a zero-divisor (resp. is always invertible) unless $(m)_{q}=0$ and also that the $q$-characteristic of $R$ is the smallest positive integer $p$ such that $(p)_{q}=0$, if it exists, and 0 otherwise.

Theorem 6.3. Assume $R$ is $q$-divisible and let $A$ be a $q$-R-algebra. Then,

1. If $q-\operatorname{char}(A)=0$, we have

$$
\mathrm{D}_{A / R, \sigma}=\overline{\mathrm{D}}_{A / R, \sigma}=\mathrm{D}_{A / R, \sigma}^{(\infty)}
$$

2. If $q-\operatorname{char}(A)=p>0$, we have

$$
\mathrm{D}_{A / R, \sigma} / \partial_{\sigma}^{p} \simeq \overline{\mathrm{D}}_{A / R, \sigma} \simeq \mathrm{D}_{A / R, \sigma}^{(\infty)} / K_{\sigma}^{[p]}
$$

where $K_{\sigma}^{[p]}$ is the free A-module generated by all $\partial_{\sigma}^{[k]}$ for $k \geq p$.

Note that with some extra conventions, assertions 1 and 2 could be turned into a single one.

Proof We use the formula from corollary 6.2

In situation 1, then all $q$-integers are invertible in $A$ and the composite map sends the canonical basis $\left\{\partial_{\sigma}^{k}\right\}_{k \in \mathbb{N}}$ of $\mathrm{D}_{\sigma}$ bijectively onto a basis of $\mathrm{D}_{\sigma}^{(\infty)}$. The first assertion follows.

In situation 2, we have $(p)_{q}=0$ in $A$ and the element $\partial_{\sigma}^{p} \in \mathrm{D}_{\sigma}$ is therefore sent to 0 . But since $R$ is $q$-divisible, we have $(m)_{q} \in R^{\times}$for $m<p$, and the composite map sends the family $\left\{\partial_{\sigma}^{k}\right\}_{k<p}$ of $\mathrm{D}_{\sigma}$ bijectively onto a basis of $\mathrm{D}_{\sigma}^{(\infty)} / K_{\sigma}^{[p]}$.

Remarks 1. The hypothesis in 1) is satisfied in the cases of classical differential equations, classical finite difference equations and classical $q$-difference equations.

More precisely, it is satisfied for example when

(a) $q=1$ and $R$ is a $\mathbb{Q}$-algebra, or

(b) $q$ is not equal to zero, not a root of 1 and belongs to a subfield $K$ of $R$.

The hypothesis in 2 is satisfied for differential equations and finite difference equations in positive characteristic as well as in the classical quantum case. More precisely, they are satisfied for example when

(a) $q=1$ and $R$ is an $\mathbb{F}_{p}$-algebra, or

(b) $q$ is a non trivial $p$-th root of 1 ( $p$ not necessarily prime) and belongs to a subfield $K$ of $R$, or

(c) $q$ is a non trivial $p$-th root of 1 with $p$ prime (but $q$ does not necessarily belong to a subfield of $R$ ).

2. Since both $\mathrm{D}_{A / R, \sigma}$ and $\mathrm{D}_{A / R, \sigma}^{(\infty)}$ commute with extensions of $R$ (although $\overline{\mathrm{D}}_{A / R, \sigma}$ does not), and $\mathrm{D}_{A / R, \sigma}$ always commutes with extensions of $A$, we can sometimes (see the remark following proposition 1.10 reduce questions to the generic case

$$
R=\mathbb{Q}(t)[s], \quad A=R[x], \quad q=t \quad \text { and } \quad h=s,
$$

and work as well over the later. In this case, thanks to the theorem, we can identify $\mathrm{D}_{A / R, \sigma}$ with $\mathrm{D}_{A / R, \sigma}^{(\infty)}$. 
3. The same proof shows that if $A$ is a twisted $R$-algebra which is only $q$-flat (but not necessarily $q$-divisible), then

(a) If $q-\operatorname{char}(A)=0$, then $\mathrm{D}_{A / R, \sigma}=\overline{\mathrm{D}}_{A / R, \sigma}$.

(b) If $q-\operatorname{char}(A)=p>0$, then $\mathrm{D}_{A / R, \sigma} / \partial_{\sigma}^{p} \simeq \overline{\mathrm{D}}_{A / R, \sigma}$.

The end of this section will be devoted to giving explicit formulas. They are usually quite formal to prove in the ring of twisted differential operators of infinite level and their analog in the twisted Weyl algebra is then easily obtained thanks to theorem 6.3 .

Proposition 6.4. We have in $\mathrm{D}_{A / R, \sigma}^{(\infty)}$,

$$
\forall k>0, \quad \partial_{\sigma}^{[k]} \circ x=q^{k} x \partial_{\sigma}^{[k]}+(k)_{q} h \partial_{\sigma}^{[k]}+\partial_{\sigma}^{[k-1]} .
$$

We have in $\mathrm{D}_{A / R, \sigma}$,

$$
\forall k>0, \quad \partial_{\sigma}^{k} \circ x=q^{k} x \partial_{\sigma}^{k}+(k)_{q}\left(h \partial_{\sigma}^{k}+\partial_{\sigma}^{k-1}\right)
$$

Proof The first assertion is simply a reformulation of proposition 4.7. For the second one, after a base change, we may reduce to the generic case 6.1 and thus assume that $R$ is $q$-divisible and $q-\operatorname{char}(R)=0$. And we may then replace $\mathrm{D}_{\sigma}$ with $\mathrm{D}_{\sigma}^{(\infty)}$ in which case we fall back onto the first equality.

Note that, in order to prove the second formula, we cannot use directly corollary 6.2 ; our equality takes place into the Weyl algebra and is not just an assertion about endomorphisms of $A$.

We concentrate now on the case $\sigma(x)=q x$.

Proposition 6.5. Assume $h=0, q \in R^{\times}$and $x \in A^{\times}$. Then, we have in $\mathrm{D}_{A / R, \sigma}^{(\infty)}$,

$$
\forall k \in \mathbb{N}, \quad \partial_{\sigma}^{[k]} \circ x^{-1}=\sum_{i=0}^{k}(-1)^{i} q^{-\frac{(2 k-i)(i+1)}{2}} x^{-i-1} \partial_{\sigma}^{[k-i]} .
$$

We have in $\mathrm{D}_{A / R, \sigma}$,

$$
\forall k \in \mathbb{N}, \quad \partial_{\sigma}^{k} \circ x^{-1}=\sum_{i=0}^{k}(-1)^{i} q^{-\frac{(2 k-i)(i+1)}{2}}(k)_{q} \cdots(k-i+1)_{q} x^{-i-1} \partial_{\sigma}^{k-i} .
$$

Proof The first assertion is a particular case of corollary 4.8 and the second one follows by the standard generic argument.

Proposition 6.6. Assume that $h=0$. Then, we have in $\mathrm{D}_{A / R, \sigma}^{(\infty)}$,

$$
\forall k, n \in \mathbb{N}, \quad \partial_{\sigma}^{[k]} \circ x^{n}=\sum_{i=0}^{k} q^{(n-i)(k-i)}\left(\begin{array}{c}
n \\
i
\end{array}\right)_{q} x^{n-i} \partial_{\sigma}^{[k-i]} .
$$

We have in $\mathrm{D}_{A / R, \sigma}$,

$$
\forall k, n \in \mathbb{N}, \quad \partial_{\sigma}^{k} \circ x^{n}=\sum_{i=0}^{k} q^{(n-i)(k-i)}(i)_{q} !\left(\begin{array}{c}
k \\
i
\end{array}\right)_{q}\left(\begin{array}{c}
n \\
i
\end{array}\right)_{q} x^{n-i} \partial_{\sigma}^{k-i} .
$$


Proof As usual, the second formula will follow from the first that we prove directly by induction on $n$. We will have

$$
\begin{aligned}
\partial_{\sigma}^{[k]} \circ x^{n}= & \sum_{i=0}^{k} q^{(n-1-i)(k-i)}\left(\begin{array}{c}
n-1 \\
i
\end{array}\right)_{q} x^{n-1-i} \partial_{\sigma}^{[k-i]} \circ x \\
= & \sum_{i=0}^{k} q^{(n-1-i)(k-i)}\left(\begin{array}{c}
n-1 \\
i
\end{array}\right)_{q} x^{n-1-i}\left(q^{k-i} x \partial_{\sigma}^{[k-i]}+\partial_{\sigma}^{[k-i-1]}\right) \\
= & \sum_{i=0}^{k} q^{(n-1-i)(k-i)}\left(\begin{array}{c}
n-1 \\
i
\end{array}\right)_{q} x^{n-1-i} q^{k-i} x \partial_{\sigma}^{[k-i]} \\
& +\sum_{i=0}^{k} q^{(n-1-i)(k-i)}\left(\begin{array}{c}
n-1 \\
i
\end{array}\right)_{q} x^{n-1-i} \partial_{\sigma}^{[k-i-1]} \\
= & \sum_{i=0}^{k} q^{(n-i)(k-i)}\left(\begin{array}{c}
n-1 \\
i
\end{array}\right)_{q} x^{n-i} \partial_{\sigma}^{[k-i]}+\sum_{i=0}^{k-1} q^{(n-i)(k-i+1)}\left(\begin{array}{c}
n-1 \\
i-1
\end{array}\right)_{q} x^{n-i} \partial_{\sigma}^{[k-i]} \\
= & \left.\sum_{i=0}^{k} q^{(n-i)(k-i)}\left(\begin{array}{c}
n-1 \\
i
\end{array}\right)_{q}+q^{n-i}\left(\begin{array}{c}
n-1 \\
i-1
\end{array}\right)_{q}\right)^{n-i} \partial_{\sigma}^{[k-i]} \\
= & \sum_{i=0}^{k} q^{(n-i)(k-i)}\left(\begin{array}{c}
n \\
i
\end{array}\right)_{q} x^{n-i} \partial_{\sigma}^{[k-i]} \cdot \square
\end{aligned}
$$

Corollary 6.7. When $h=0$, we have, for all $n \in \mathbb{N}$,

$$
\partial_{\sigma}^{[k]}\left(x^{n}\right)=\left\{\begin{array}{cl}
\left(\begin{array}{l}
n \\
k
\end{array}\right)_{q} x^{n-k} & \text { if } n \geq k \\
0 & \text { otherwise }
\end{array}\right.
$$

and

$$
\partial_{\sigma}^{k}\left(x^{n}\right)=\left\{\begin{array}{cl}
(n)_{q} \cdots(n-k+1)_{q} x^{n-k} & \text { if } n \geq k \\
0 & \text { otherwise }
\end{array} .\right.
$$

Proof The first assertion follows from the proposition and the second one uses the inclusion $\bar{D}_{A / R, \sigma} \subset D_{A / R, \sigma}^{(\infty)}$.

Corollary 6.8. Assume that $h=0$. Then, we have

$$
\forall n \in \mathbb{N} \backslash\{0\}, \quad \partial_{\sigma}\left(x^{n}\right)=(n)_{q} x^{n-1} .
$$

If $x \in A^{\times}$and $q \in R^{\times}$, then the formula actually holds for all $n \in \mathbb{Z}$.

Proof Only the second part needs a proof: if $x$ is invertible, we have for $n>0$

$$
\begin{aligned}
0 & =\partial_{\sigma}(1)=\partial_{\sigma}\left(x^{n} x^{-n}\right)=\partial_{\sigma}\left(x^{n}\right) x^{-n}+\sigma\left(x^{n}\right) \partial_{\sigma}\left(x^{-n}\right) \\
& =(n)_{q} x^{n-1} x^{-n}+q^{n} x^{n} \partial_{\sigma}\left(x^{-n}\right)=(n)_{q} x^{-1}+q^{n} x^{n} \partial_{\sigma}\left(x^{-n}\right),
\end{aligned}
$$

from which we derive, when $q \in R^{\times}$,

$$
\partial_{\sigma}\left(x^{-n}\right)=-q^{-n}(n)_{q} x^{-n-1}=(-n)_{q} x^{-n-1}
$$

\section{Formal deformations of twisted differential operators}

In this section, we study the relation between twisted differential operators relative to various endomorphisms of $A$. We are particularly interested in the comparison of our twisted differential 
operators with usual differential operators. We assume that there exists a twisted coordinate $x$, that we fix for the rest of the section, and we write $y_{\sigma}:=x-\sigma(x)$.

Recall that the ring $\mathrm{D}_{\sigma}^{(\infty)}$ of differential operators (of infinite level) comes with a natural increasing filtration by $A$-submodules

$$
\mathrm{D}_{\sigma}^{(\infty)}=\cup_{m=0}^{\infty} \operatorname{Diff}_{m, \sigma}(A),
$$

which is called the order filtration. The choice of the twisted coordinate determines a splitting of this filtration. To see this, we let for all $m \in \mathbb{N}, K_{\sigma}^{[m]} \subset \mathrm{D}_{\sigma}^{(\infty)}$ be the free $A$-module generated by all $\partial_{\sigma}^{[k]}$ for $k \geq m$. Note that this is actually a filtration by left ideals.

Definition 7.1. The decreasing filtration by the $K_{\sigma}^{[m]}$ is called the ideal filtration on $\mathrm{D}_{\sigma}^{(\infty)}$. The module of twisted differential operators of infinite level and infinite order on $A$ is

$$
\widehat{\mathrm{D}}_{A / R, \sigma}^{(\infty)}=\lim _{\longleftarrow} \mathrm{D}_{\sigma}^{(\infty)} / K_{\sigma}^{[m+1]} .
$$

We might again drop the index $A / R$ and write $\widehat{\mathrm{D}}_{\sigma}^{(\infty)}$. The decreasing filtration of $\widehat{\mathrm{D}}_{\sigma}^{(\infty)}$ by the closures $\widehat{K}_{\sigma}^{[m]}$ of the $K_{\sigma}^{[m]}$ 's will also be called the ideal filtration .

Remarks 1. The ideal filtration is separated, which means that we have $\cap_{m} K_{\sigma}^{[m+1]}=\{0\}$ and it follows that $\mathrm{D}_{\sigma}^{(\infty)} \subset \widehat{\mathrm{D}}_{\sigma}^{(\infty)}$. Actually, any $\varphi \in \widehat{\mathrm{D}}_{\sigma}^{(\infty)}$ can be uniquely written as an infinite sum $\sum_{0}^{\infty} z_{k} \partial_{\sigma}^{[k]}$ with $z_{k} \in A$ (and conversely). In other words, we have the isomorphisms of $A$-modules

$$
\mathrm{D}_{\sigma}^{(\infty)}=\bigoplus_{k \in \mathbb{N}} A \partial_{\sigma}^{[k]} \text { and } \widehat{\mathrm{D}}_{\sigma}^{(\infty)}=\prod_{k \in \mathbb{N}} A \partial_{\sigma}^{[k]}
$$

2. The ideal filtration defines a splitting of the order filtration in the sense that

$$
\mathrm{D}_{\sigma}^{(\infty)}=\operatorname{Diff}_{m, \sigma}(A) \oplus K_{\sigma}^{[m+1]} \quad \text { and } \quad \widehat{\mathrm{D}}_{\sigma}^{(\infty)}=\operatorname{Diff}_{m, \sigma}(A) \oplus \widehat{K}_{\sigma}^{[m+1]}
$$

as $A$-modules.

3. $\widehat{\mathrm{D}}_{\sigma}^{(\infty)}$ is not a ring in general. More precisely, multiplication on $\mathrm{D}_{\sigma}^{(\infty)}$ is not continuous for the ideal filtration: we always have $\partial_{\sigma}^{[m]} \rightarrow 0$ when $m \rightarrow \infty$ but, if $\sigma(x)=q x$ and $x \in A^{\times}$for example, we can see that

$$
\forall m \in \mathbb{N}, \quad \partial_{\sigma}^{[m]} \circ x^{-1} \equiv \partial_{\sigma}^{[m]}\left(x^{-1}\right)=x^{-m-1} \neq 0 \quad \bmod K_{\sigma}^{[1]} .
$$

If $A$ is any ring and $A[\xi]$ denotes the polynomial ring in one variable $\xi$, then the natural filtration on $\operatorname{Hom}_{A}(A[\xi], A)$ is the decreasing filtration by the kernels of the surjections

$$
\operatorname{Hom}_{A}(A[\xi], A) \rightarrow \operatorname{Hom}_{A}\left(A[\xi]_{\leq m}, A\right),
$$

where $A[\xi]_{\leq m}$ denotes as before the $A$-module of polynomials of degree at most $m$. The corresponding topology will be called the natural topology of $\operatorname{Hom}_{A}(A[\xi], A)$. Note that $\operatorname{Hom}_{A}(A[\xi], A)$ is separated and complete for the natural topology:

$$
\lim _{\longleftarrow} \operatorname{Hom}_{A}\left(A[\xi]_{\leq m}, A\right)=\operatorname{Hom}_{A}\left(\lim _{\longrightarrow} A[\xi]_{\leq m}, A\right)=\operatorname{Hom}_{A}(A[\xi], A) .
$$

Lemma 7.2 (Formal density). The map

$$
\begin{aligned}
A[\xi] & \longrightarrow P_{A / R} \\
\xi \longmapsto & \sim \widetilde{x}-x
\end{aligned}
$$

induces by duality an isomorphism of topological A-modules

$$
\widehat{\mathrm{D}}_{A / R, \sigma}^{(\infty)} \stackrel{\simeq}{\longrightarrow} \operatorname{Hom}_{A}(A[\xi], A)
$$

More precisely, the ideal filtration corresponds to the natural filtration. 
Proof It follows from corollary 1.13 that the cokernel of the map

$$
A[\xi]_{\leq m} \longleftrightarrow A[\xi] \longrightarrow P \longrightarrow P_{(n)_{\sigma}}
$$

is generated by $\xi^{(k)}$ for $m<k \leq n$. Moreover, this map is injective when $m \leq n$. Dually, it means that the corresponding map

$$
\operatorname{Diff}_{n, \sigma}(A) \simeq \operatorname{Hom}_{A}\left(P_{(n)_{\sigma}}, A\right) \longrightarrow \operatorname{Hom}_{A}\left(A[\xi]_{\leq m}, A\right)
$$

is surjective for $m \leq n$ and that its kernel is exactly $K_{\sigma}^{[m+1]} \cap \operatorname{Diff}_{n, \sigma}(A)$ (it is generated by $\partial_{\sigma}^{[k]}$ for $n \geq k>m$ ). Taking direct limits on the left, we see that the map

$$
\mathrm{D}_{\sigma}^{(\infty)} \longrightarrow \operatorname{Hom}_{A}\left(A[\xi]_{\leq m}, A\right)
$$

is surjective with kernel exactly $K_{\sigma}^{[m+1]}$. In other words, we get a canonical isomorphism of $A$ modules

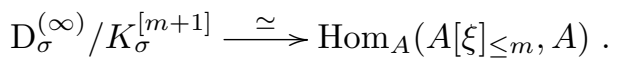

Thus, taking inverse limits on both sides gives the result.

Remarks 1. By construction, there exists a commutative diagram

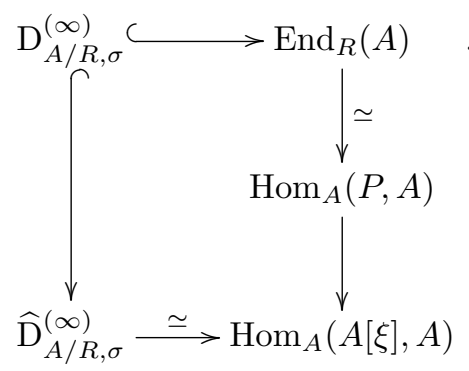

2. We will usually denote by $\tilde{\varphi} \in \operatorname{Hom}_{A}(A[\xi], A)$ the image of $\varphi \in \widehat{\mathrm{D}}_{A / R, \sigma}^{(\infty)}$. This is compatible with the previous notation for linearization. In particular, we have

$$
\forall k, n \in \mathbb{N}, \quad \widetilde{\partial_{\sigma}^{[k]}}\left(\xi^{(n)}\right)=\left\{\begin{array}{ll}
1 & \text { if } k=n \\
0 & \text { otherwise }
\end{array} .\right.
$$

3. When $A=R[x]$, we actually get

$$
\mathrm{D}_{A / R, \sigma}^{(\infty)} \hookrightarrow \widehat{\mathrm{D}}_{A / R, \sigma}^{(\infty)} \stackrel{\simeq}{\longrightarrow} \operatorname{Hom}_{A}(A[\xi], A) \stackrel{\simeq}{\longrightarrow} \operatorname{Hom}_{A}(P, A) \stackrel{\simeq}{\longrightarrow} \operatorname{End}_{R}(A)
$$

and $\widehat{D}_{A / R, \sigma}^{(\infty)}$ is a ring in this very special case.

4. There exists a complex analytic analog of the density lemma (for usual differential operators) as explained by Zogman Mebkhout and Luis Narváez in [25]: the ring of algebraic differential operators is dense in the ring of continuous endomorphisms of the structural sheaf.

Although it is difficult to give an explicit description of the isomorphism (7.1), we can at least show the following:

Lemma 7.3. We have

$$
\forall n \in \mathbb{N} \backslash\{0\}, \quad \tilde{\partial}_{\sigma}\left(\xi^{n}\right)=(\sigma(x)-x)^{n-1} .
$$

More generally, if $\tau$ is another R-algebra endomorphism of $A$, we have

$$
\forall n \in \mathbb{N} \backslash\{0\}, \quad \tilde{\partial}_{\sigma}\left(\xi^{(n)_{\tau}}\right)=\prod_{i=1}^{n-1}\left(\sigma(x)-\tau^{i}(x)\right),
$$

where $\xi^{(n)_{\tau}}:=\xi \tau(\xi) \cdots \tau^{n-1}(\xi)$ denotes the twisted power with respect to the endomorphism $\tau$. 
Proof By definition, $\tilde{\partial}_{\sigma}$ is the unique $A$-linear function on $A[\xi]$ such that $\tilde{\partial}_{\sigma}\left(\xi^{(n)}\right)=1$ for $n=1$ and 0 otherwise. We consider now the unique $A$-linear function $u$ on $A[\xi]$ such that $u\left(\xi^{n}\right)=(\sigma(x)-x)^{n-1}$ for $n>0$ and $u(1)=0$. We want to show that $u=\tilde{\partial}_{\sigma}$. The map $u$ may be seen as the composition of division by $\xi$ on $A[\xi]$ (after removing the constant term) and evaluation at $\sigma(x)-x$. But we have

$$
\xi^{(n)}:=\xi \sigma(\xi) \cdots \sigma^{n-1}(\xi),
$$

and we know that $\sigma(\xi)=\xi+x-\sigma(x)$. Therefore, it is clear that for $n \geq 2$, we will get $u\left(\xi^{(n)}\right)=0$. Of course, for $n=0$ we have $\xi^{(n)}=1$ and we also obtain 0 . Finally, for $n=1$, we have $\xi^{(n)}=\xi$ and we get 1 .

The proof of the second formula follows the same lines. From the first part, we can interpret $\tilde{\partial}_{\sigma}$ as the composition of division by $\xi$ on $A[\xi]$ and evaluation at $\sigma(x)-x$. We want to apply this to $\xi^{(n)_{\tau}}=\prod_{i=0}^{n-1} \tau^{i}(\xi)$ and we know that $\tau^{i}(\xi)=\xi+x-\tau^{i}(x)$. Thus, we see that

$$
\tilde{\partial}_{\sigma}\left(\xi^{(n)_{\tau}}\right)=\prod_{i=1}^{n-1}\left(\sigma(x)-x+x-\tau^{i}(x)\right)=\prod_{i=1}^{n-1}\left(\sigma(x)-\tau^{i}(x)\right) .
$$

We can actually derive a remarkable consequence of the density lemma (recall that when $\tau$ is an endomorphism of the $R$-algebra $A$, we call $\tau$-coordinate any twisted coordinate relative to $\tau$ ):

Proposition 7.4 (Formal deformation). If $x$ is a also a $\tau$-coordinate for another $R$-endomorphism $\tau$ of $A$, then there exists a canonical isomorphism of topological A-modules (that depends on $x$ )

$$
\widehat{\mathrm{D}}_{A / R, \sigma}^{(\infty)} \stackrel{\simeq}{\longrightarrow} \widehat{\mathrm{D}}_{A / R, \tau}^{(\infty)}
$$

More precisely, it is compatible with the ideal filtrations.

Recall that the hypothesis is always satisfied when $A$ is a $\tau$-twisted localization of $R[x]$.

Proof We may just compose the isomorphism of the formal density lemma 7.2 with the inverse of the analogous isomorphism for $\tau$.

When the coordinate $x$ is fixed, we may (and will) identify these two topological (or even, filtered) $A$-modules. It is worth mentioning a particular case of this corollary that is of great interest (this is the case $\tau=\operatorname{Id}_{A}$ in which case we say usual coordinate instead of $\tau$-coordinate):

Corollary 7.5. Assume that $x$ is not only a $\sigma$-coordinate, but also a usual coordinate on A. Then, there exists a canonical isomorphism

$$
\widehat{\mathrm{D}}_{A / R, \sigma}^{(\infty)} \stackrel{\simeq}{\longrightarrow} \widehat{\mathrm{D}}_{A / R}^{(\infty)}
$$

Note that the hypothesis is always satisfied when $A$ is a localization of $R[x]$. More generally, when $A$ is smooth over $R$, a usual coordinate is the same thing as an étale coordinate.

Remark 1. In theorem 2 of the introduction to [27], Pulita shows that, in the $p$-adic world, some differential modules have a natural structure of $\sigma$-module. The main idea is to realize a formal solution of the differential module as formal solution of some $\sigma$-module. This is analog to the way we derive the formal deformation theorem from the formal density lemma. It seems that the first result in this direction was obtained by André and Di Vizio in [3].

2. In [16], Michel Gros and the first author were able to prove a quantum Simpson's correspondence. Assume $R=\mathbb{Z}[q]$, where $q$ is a $p$-th root of unity with $p$ prime, $A$ is the polynomial ring and $\sigma(x)=q x$. Then, the category of $A$-modules endowed with a quasi-nilpotent $q$-derivation is equivalent to the category of $A$-modules endowed with an $A$-linear quasi-nilpotent endomorphism. This is derived from an isomorphism quite analog to (7.1) which reads

$$
\widehat{D}_{q} \simeq \operatorname{End}_{\widehat{Z}_{q}}\left(\widehat{\mathrm{Z}_{q} A}\right) \text {. }
$$


3. We want to insist on the fact that the isomorphism of corollary 7.5 is not an isomorphism of rings. Actually, neither side of this isomorphism is a ring. In order to achieve this goal, it would be necessary to refine the topology. After the remarks following corollary 7.7, we will give some elementary but non-trivial examples over the complex numbers, and refer the reader to forthcoming articles for a detailed study of the ultrametric and the $q$-characteristic zero cases.

We give now some explicit formulas in order to express the twisted derivation from one world as a twisted differential operators in another world:

Proposition 7.6. Assume that $x$ is a also a $\tau$-coordinate for some other $R$-endomorphism $\tau$ of A. Then, we have

$$
\partial_{\sigma}=\sum_{k=1}^{\infty}\left(\prod_{i=1}^{k-1}\left(\sigma(x)-\tau^{i}(x)\right)\right) \partial_{\tau}^{[k]}
$$

Proof This follows directly by duality from lemma 7.3

Corollary 7.7. Assume that $x$ is also a usual coordinate on A. Then,

$$
\partial_{\sigma}=\sum_{k=1}^{\infty}(\sigma(x)-x)^{k-1} \partial^{[k]}
$$

and

$$
\sigma=\sum_{k=0}^{\infty}(\sigma(x)-x)^{k} \partial^{[k]}
$$

Proof The first assertion follows directly from the proposition and the other one is deduced from the equality $\sigma=1-y_{\sigma} \partial_{\sigma}$.

Remarks 1. In the case $\sigma(x)=q x$, the formulas read

$$
\partial_{\sigma}=\sum_{k=1}^{\infty}(q-1)^{k-1} x^{k-1} \partial^{[k]} \quad \text { and } \quad \sigma=\sum_{k=0}^{\infty}(q-1)^{k} x^{k} \partial^{[k]} \text {. }
$$

2. In the case $\sigma(x)=x+h$, the formulas read

$$
\partial_{\sigma}=\sum_{k=1}^{\infty} h^{k-1} \partial^{[k]} \quad \text { and } \quad \sigma=\sum_{k=0}^{\infty} h^{k} \partial^{[k]} .
$$

Examples 1. Consider the differential equation

$$
\frac{\mathrm{d} s}{\mathrm{~d} z}=s
$$

over the complex numbers. If we use the second formula of 7.2 , we see that the corresponding $q$-difference equation is given by

$$
s(q z)=e^{(q-1) z} s(z) .
$$

If we use 7.3 instead, we obtain the difference equation

$$
s(z+h)=e^{h} s(z) .
$$


2. Consider now the differential equation

$$
\frac{\mathrm{d} s}{\mathrm{~d} z}=\frac{a}{z} s
$$

for some $a \in \mathbb{C}$. Then, the $q$-difference equation will be given by

$$
s(q z)=q^{a} s(z)
$$

and the difference equation is

$$
s(z+h)=\left(\frac{z+h}{z}\right)^{a} s(z) .
$$

We may also go the other way, and express the usual derivation in term of twisted derivations. We only do the classical cases:

Corollary 7.8. Assume that $x$ is also a usual coordinate on A. Then,

1. If $\sigma(x)=q x$, we have

$$
\partial=\sum_{k=1}^{\infty}(1-q)^{k-1}(k-1)_{q} ! x^{k-1} \partial_{\sigma}^{[k]} .
$$

2. If $\sigma(x)=x+h$, we have

$$
\partial=\sum_{k=1}^{\infty}(-1)^{k-1} h^{k-1}(k-1) ! \partial_{\sigma}^{[k]} .
$$

Proof In the formula of proposition 7.6 we replace $\tau$ by $\sigma$ and $\sigma$ by Id respectively, in order to obtain

$$
\begin{aligned}
\partial & =\sum_{k=1}^{\infty} \prod_{i=1}^{k-1}\left(x-q^{i} x\right) \partial_{\sigma}^{[k]}=\sum_{k=1}^{\infty} \prod_{i=1}^{k-1}\left(1-q^{i}\right) x^{k-1} \partial_{\sigma}^{[k]} \\
& =\sum_{k=1}^{\infty}(1-q)^{k-1} \prod_{i=1}^{k-1}(i)_{q} x^{k-1} \partial_{\sigma}^{[k]}=\sum_{k=1}^{\infty}(1-q)^{k-1}(k-1)_{q} ! x^{k-1} \partial_{\sigma}^{[k]}
\end{aligned}
$$

in the first case, and

$$
\partial=\sum_{k=1}^{\infty} \prod_{i=1}^{k-1}(-i h) \partial_{\sigma}^{[k]}=\sum_{k=1}^{\infty}(-h)^{k-1}(k-1) ! \partial_{\sigma}^{[k]}
$$

in the other one.

We may also apply proposition 7.6 in order to make more precise the statement of proposition 4.13

Corollary 7.9. Assume that $x$ is also a $\sigma^{m}$-coordinate for $A$ over $R$, then we have

$$
\partial_{\sigma^{m}}=\sum_{k=1}^{m}\left(\prod_{i=1}^{k-1}\left(\sigma^{m}(x)-\sigma^{i}(x)\right)\right) \partial_{\sigma}^{[k]} .
$$

One can give a more concrete formula in the quantum situation:

Corollary 7.10. Assume that $x$ is also a $\sigma^{m}$-coordinate for $A$ over $R$ and that $\sigma(x)=q x$. Then, we have

$$
\partial_{\sigma^{m}}=\sum_{k=1}^{m} q^{\frac{k(k-1)}{2}}(q-1)^{k-1}(m-1)_{q} \cdots(m-k+1)_{q} x^{k-1} \partial_{\sigma}^{[k]} .
$$


Proof We compute the coefficient of $\partial_{\sigma}^{[k]}$ for $1 \leq k \leq m$ :

$$
\begin{aligned}
\prod_{i=1}^{k-1}\left(q^{m} x-q^{i} x\right) & =\left(\prod_{i=1}^{k-1} q^{i}\right)\left(\prod_{i=1}^{k-1}\left(q^{m-i}-1\right)\right) x^{k-1} \\
& =q^{\frac{k(k-1)}{2}}(q-1)^{k-1}\left(\prod_{i=1}^{k-1}(m-i)_{q}\right) x^{k-1}
\end{aligned}
$$

Example We will have $\partial_{\sigma^{2}}=\partial_{\sigma}+q(q-1) x \partial_{\sigma}^{[2]}$.

\section{Formal confluence}

We explain here how one can use the quantum Weyl algebra in order to approximate a usual differential operator. We assume that $A$ is a quantum $R$-algebra which means that we are given a twisted coordinate $x$ on $A$ with $\sigma(x)=q x+h$ with $q, h \in R$.

Recall from $\left[23\right.$ that the twisted Weyl $R$-algebra $\mathrm{D}_{\sigma}$ has an increasing filtration by free $A$-modules of finite rank

$$
\mathrm{Fil}^{m} \mathrm{D}_{\sigma}=\bigoplus_{k \leq m} A \partial_{\sigma}^{k}
$$

that is called the order filtration. But it also has a decreasing filtration by free $A$-modules (of infinite rank)

$$
K_{\sigma}^{m}=\bigoplus_{k \geq m} A \partial_{\sigma}^{k}
$$

that we called the ideal filtration. We will consider the completion

$$
\widehat{\mathrm{D}}_{\sigma}=\lim _{\leftarrow} \mathrm{D}_{\sigma} / K_{\sigma}^{m+1}
$$

that also comes with its ideal filtration and we have $\mathrm{D}_{\sigma} \subset \widehat{\mathrm{D}}_{\sigma}$. Note that $\widehat{\mathrm{D}}_{\sigma}$ is the set of all infinite sums $\sum_{0}^{\infty} z_{k} \partial_{\sigma}^{k}$ with $z_{k} \in A$. In other words, there exists an isomorphism of $A$-modules

$$
\widehat{\mathrm{D}}_{\sigma}=\prod_{k \in \mathbb{N}} A \partial_{\sigma}^{k} .
$$

The $A$-module $\widehat{\mathrm{D}}_{\sigma}$ is not a ring in general. However, the result holds in the finite quantum characteristic case as we can check right now:

Proposition 8.1. If $A$ is $q$-flat and $q-\operatorname{char}(A)=p>0$, then multiplication is continuous for the ideal topology on $\mathrm{D}_{A / R, \sigma}$ and turns $\widehat{\mathrm{D}}_{A / R, \sigma}$ into an $R$-algebra.

Proof From the equality (see the remark following theorem 6.3

$$
\mathrm{D}_{\sigma} / \partial_{\sigma}^{p} \simeq \overline{\mathrm{D}}_{\sigma}
$$

and the fact that $\overline{\mathrm{D}}_{\sigma}$ is a ring, we deduce that the (left) ideal generated by $\partial_{\sigma}^{p}$ is a two sided ideal. Since multiplication is automatically continuous for the $\partial_{\sigma}^{p}$-adic filtration, it follows that it is also continuous for the ideal filtration (which is the filtration by left ideals generated by the powers of $\left.\partial_{\sigma}\right)$.

Lemma 8.2. The composite map

$$
D_{A / R, \sigma} \longrightarrow \overline{\mathrm{D}}_{A / R, \sigma} \longleftrightarrow \mathrm{D}_{A / R, \sigma}^{(\infty)}
$$

is compatible with the ideal filtrations. Moreover, if $R$ is $q$-divisible and $q$-char $(R)=0$, then $\widehat{\mathrm{D}}_{A / R, \sigma} \simeq \widehat{\mathrm{D}}_{A / R, \sigma}^{(\infty)}$. 
This applies in particular when $R$ is a $\mathbb{Q}$-algebra and $\sigma=\mathrm{Id}$.

Proof The first assertion follows from corollary 6.2 and the second from theorem 6.3 .

We can now state our fist confluence theorem (recall that a usual coordinate is the same thing as an étale coordinate when $A$ is smooth over $R$ ):

Theorem 8.3 (Formal quantum confluence 1). Let $R$ be a $\mathbb{Q}$-algebra, $A$ a $q$-R-algebra for some $q \in R$. Assume that the quantum coordinate on $A$ is also a usual coordinate. Then, there exists a canonical map of filtered A-modules

$$
\mathrm{D}_{A / R, \sigma} \rightarrow \widehat{\mathrm{D}}_{A / R}
$$

Moreover, if $R$ is q-divisible, we have

1. If $q-\operatorname{char}(A)=0$, then the map 8.1 is injective and $\mathrm{D}_{A / R, \sigma}$ is dense in $\widehat{\mathrm{D}}_{A / R}$. Actually, the inclusion morphism $\mathrm{D}_{A / R, \sigma} \subset \widehat{\mathrm{D}}_{A / R}$ is strict for the ideal filtrations in the sense that $\mathrm{D}_{A / R, \sigma} \cap \widehat{K}^{m}=K_{\sigma}^{m}$ for all $m \in \mathbb{N}$.

2. If $q-\operatorname{char}(A)=p>0$, then the map (8.1) induces an isomorphism

$$
\left(\mathrm{D}_{A / R, \sigma} / \partial_{\sigma}^{p} \simeq\right) \quad \overline{\mathrm{D}}_{A / R, \sigma} \simeq \mathrm{D}_{A / R} / \partial^{p} .
$$

Proof The map (8.1) is simply the composite

$$
\mathrm{D}_{\sigma} \rightarrow \mathrm{D}_{\sigma}^{(\infty)} \hookrightarrow \widehat{\mathrm{D}}_{\sigma}^{(\infty)} \simeq \widehat{\mathrm{D}}^{(\infty)} \simeq \widehat{\mathrm{D}}_{A / R}
$$

where the next to the last map is the formal deformation isomorphism of corollary 7.5 and the last one comes from lemma 8.2 applied to the case $\sigma=$ id since $R$ is a $\mathbb{Q}$-algebra.

If we assume that $R$ is $q$-divisible and that $q-\operatorname{char}(A)=0$, then lemma 8.2 tells us that $\mathrm{D}_{\sigma}=\mathrm{D}_{\sigma}^{(\infty)}$ as filtered rings (for the ideal filtrations) and we can use corollary 7.5 again.

Finally, when $R$ is $q$-divisible but $q-\operatorname{char}(A)=p>0$, then $(p)_{q}=0$ in $A$ and all $(m)_{q} \in R^{\times}$for $m<p$. We can use the last assertion of theorem 6.3 and the fact that the isomorphism of corollary 7.5 is strictly compatible with the filtrations.

As in [23, we denote by $A[T]_{\sigma}$ the twisted polynomial ring associated to $\sigma$ : this is the non commutative polynomial ring in $T$ over $A$ with the commutation rule $T x=\sigma(x) T$. Recall also that the twisted coordinate $x$ is said to be strong if $x-\sigma(x) \in A^{\times}$.

Corollary 8.4. If $R$ is a $q$-divisible $\mathbb{Q}$-algebra, $q$-char $(A)=0, x$ is strong and is also a $\sigma^{n}$ coordinate for all $n \in \mathbb{N}$, then, the A-linear map

$$
\begin{aligned}
A[T]_{\sigma} \longrightarrow & \widehat{\mathrm{D}}_{A / R} \\
T^{n} \longmapsto & \sum_{k=0}^{\infty} \frac{1}{k !}\left(\sigma^{n}(x)-x\right)^{k} \partial^{k}
\end{aligned}
$$

has dense image.

Proof Compose the isomorphism of theorem 6.13 of 23 . with the map of theorem 8.3 The formula comes from corollary 7.7

Example The theorem (and its corollary) applies in particular when $q$ is not a root of unity in some field $K$ of characteristic zero, $R$ is an algebra containing $K, A=R\left[x, x^{-1}\right]$ and $\sigma(x)=q x$. In this situation, $\mathrm{D}:=\mathrm{D}_{A / R}$ is the non commutative ring $R\left[x, x^{-1}, \partial\right]$ with the commutation rule $\partial x=x \partial+1$ and we may see $\widehat{\mathrm{D}}$ (which is not a ring) as the set of infinite sums $R\left[x, x^{-1}\right][[\partial]]$. Also, 
$\mathrm{D}_{\sigma}:=\mathrm{D}_{A / R, \sigma}$ is the non commutative ring $R\left[x, x^{-1}, \partial_{\sigma}\right]$ with the commutation rule $\partial_{\sigma} x=q x \partial_{\sigma}+1$. The map of the theorem satisfies

$$
\partial_{\sigma} \mapsto \sum_{k=1}^{\infty} \frac{1}{k !}(q-1)^{k-1} x^{k-1} \partial^{k}
$$

and the map of the corollary is given by

$$
T^{n} \mapsto \sum_{k=0}^{\infty} \frac{1}{k !}\left(q^{n}-1\right)^{k} x^{k} \partial^{k} .
$$

In the next section, we will have to move between $\sigma$ and powers of $\sigma$ (or more precisely, the other way around, between $\sigma$ and roots of $\sigma$ ).

Proposition 8.5. Let $m \in \mathbb{N} \backslash\{0\}$. If $(m)_{q} ! \in R^{\times}$, then there exists a unique A-linear ring homomorphism

$$
\begin{aligned}
\mathrm{D}_{A / R, \sigma^{m}} & \iota_{\sigma, m} \\
\partial_{\sigma^{m}} \longmapsto & \mathrm{D}_{A / R, \sigma} \\
\longrightarrow & \sum_{k=1}^{m} \frac{1}{(k)_{q} !}\left(\prod_{i=1}^{k-1}\left(\sigma^{m}(x)-\sigma^{i}(x)\right)\right) \partial_{\sigma}^{k}
\end{aligned}
$$

Moreover, the diagram

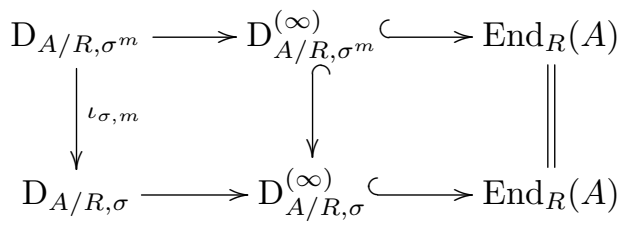

is commutative.

Proof We may assume (see the second remark following theorem 6.3 that we are in the generic situation

$$
R=\mathbb{Q}(t)[s], \quad A=R[x], \quad q=t \quad \text { and } h=s .
$$

But then, the left horizontal arrows in diagram (8.3) are bijective. In other words, we can identify the twisted Weyl $R$-algebras with the rings of twisted differential operators of infinite level and use corollary 7.9 and corollary 6.2

Remarks 1. In the case $h=0$, we have thanks to corollary 7.10 the more concrete formula:

$$
\partial_{\sigma^{m}} \longmapsto \sum_{k=1}^{m} \frac{q^{\frac{k(k-1)}{2}}(q-1)^{k-1}}{(k)_{q}}\left(\begin{array}{c}
m-1 \\
k-1
\end{array}\right)_{q} x^{k-1} \partial_{\sigma}^{k}
$$

2. Assume that $R$ is $q$-divisible. If $q-\operatorname{char}(R)=p>0$, then the hypothesis is satisfied if and only if $m<p$. Of course if $q-\operatorname{char}(R)=0$, then the hypothesis is satisfied for any $m \in \mathbb{N}$.

3. Just to give an idea of the geometric intuition, the geometric counterpart of the twisted localization hypothesis should be the requirement to work on a Zariski open subset of "the quantum line".

Proposition 8.6. Let $m, n \in \mathbb{N} \backslash\{0\}$. If $(m n)_{q}$ ! is invertible, then

$$
\iota_{\sigma, m n}=\iota_{\sigma^{m}, n} \circ \iota_{\sigma, m}
$$

Proof After a base change, we may assume that all $q$-integers are invertible and identify twisted Weyl $R$-algebras with rings of twisted differential operators of infinite level. The assertion then becomes a triviality. 
Proposition 8.7. If $(m)_{q}$ ! is invertible in $R$, then the diagram
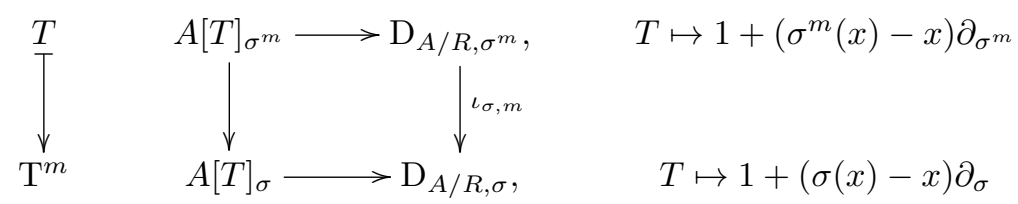

is commutative.

Proof Again, we may assume that all $q$-integers are invertible in $R$ and use the second remark following theorem 6.13 of $[23$.

\section{Formal confluence in positive quantum characteristic}

In this section, we extend the formal confluence theorem to the case of positive quantum characteristic. In order to do that, it is necessary to use the $S$-twisted theory where $S$ a filtering (for division) set of positive integers. Thus, we assume here that $A$ is an $S$-twisted $R$-algebra in the sense of [23] (it is endowed with a compatible system of $n$-th roots $\sigma_{n}$ of $\sigma$ for all $n \in S$ ).

We recall from [22] that a system of roots of $q \in R$ is a compatible family $q:=\left\{q_{n}\right\}_{n \in S}$ of $n$-th roots of $q$. We call the system $\underline{q}$ admissible if

$$
\forall n \in S, \quad(n)_{q^{n}} \in R^{\times} .
$$

This is a natural condition in order to define the q-rational number $(r)_{q}$ for any $r \in \mathbb{N} \frac{1}{S}$. More precisely, if $r=\frac{m}{n}$ with $m \in \mathbb{N}$ and $n \in S$, then,

$$
(r)_{q}:=\frac{(m)_{q_{n}}}{(n)_{q_{n}}}
$$

only depends on $r$ and not on the choice of $m$ and $n$. It is convenient to introduce the following terminology:

Definition 9.1. Let $\left\{q_{n}\right\}_{n \in S}$ be a system of roots of $q$ in $R$. Then, $R$ is said to be $q$-divisible if $R$ is $q_{n}$-divisible for all $n \in S$.

When the system is admissible, there exists a nice equivalent definition:

Lemma 9.2. Let $\left\{q_{n}\right\}_{n \in S}$ be an admissible system of roots of $q$. Then $R$ is $\underline{q}$-divisible if and only if

$$
\forall r \in \mathbb{N} \frac{1}{S}, \quad(r)_{q} \in R^{\times} \cup\{0\} .
$$

Proof If $r=\frac{m}{n}$ with $m \in \mathbb{N}$ and $n \in S$, we have $(r)_{q}=\frac{(m)_{q_{n}}}{(n)_{q_{n}}}$. It follows that $(r)_{q} \in R^{\times}$(resp. $=0)$ if and only if $(m)_{q_{n}} \in R^{\times}($resp. $=0)$.

Remarks 1. If $1-q \in R^{\times}$, then $q$ is admissible. In particular, if $q \in K$ where $K$ is a subfield of $R$ and $q \neq 0$, then $\underline{q}$ is admissible.

2. If $\underline{q} \subset K$ where $K$ is a subfield of $R$, then $R$ is $\underline{q}$-divisible.

Lemma 9.3. For a system $q:=\left\{q_{n}\right\}_{n \in S}$ of roots $q$, the following are equivalent:

1. $\underline{q}$ is admissible and for all $r \in \mathbb{N} \frac{1}{S} \cap[0,1]$, we have $(r)_{q} \in R^{\times}$.

2. For all $n \in S$, we have $(n)_{q_{n}} ! \in R^{\times}$. 
Proof First of all, the second condition also implies that $q$ is admissible and we may therefore make this assumption. If $r=\frac{m}{n}$ with $m \in \mathbb{N}$ and $n \in S$, we know that $(r)_{q} \in R^{\times}$if and only if $(m)_{q_{n}} \in R^{\times}$. Also, we have $r \leq 1$ if and only $m \leq n$. Thus, the second condition, which means that $(m)_{q_{n}} \in R^{\times}$whenever $m \leq n$, is equivalent to the requirement that $(r)_{q} \in R^{\times}$for $r \leq 1$.

Definition 9.4. Such a system of roots will be called strongly admissible.

Lemma 9.5. If $\underline{q}$ is an admissible (resp. a strongly admissible) system of roots, and we write for all $n \in S, p_{n}:=\bar{q}_{n}-\operatorname{char}(R)$, then we have

$$
\forall n \in S, \quad p_{n} \nmid n\left(\text { resp. } p_{n}>n\right) \text { or } \quad p_{n}=0 .
$$

When $R$ is $\underline{q}$-divisible, the converse is true.

Proof By definition, $q_{n}$ is admissible (resp. strongly admissible) if and only if $(n)_{q_{n}} \in R^{\times}$(resp. $\left.(n)_{q_{n}} ! \in R^{\times}\right)$. By definition also, we have $p_{n} \neq 0$ and $p_{n} \mid n$ (resp. $\left.p_{n} \leq n\right)$ if and only if $(n)_{q_{n}}=0$ (resp. $\left.(n)_{q_{n}} !=0\right)$. We see that these two conditions are mutually exclusive in general and that they are exhaustive when $R$ is $q_{n}$-divisible.

There usually exists strongly admissible systems as the next result shows:

Proposition 9.6. Assume that $R$ is $\underline{q}$-divisible and that $q-\operatorname{char}(R)=p>0$. Then, if $\underline{q}$ is a system of $p^{n}$-th root of $q$, it is strongly admissible.

Proof It follows from proposition 1.16 of [22] that for all $n \in S$, we have $q_{p^{n}}-\operatorname{char}(R)=p^{n+1}>p^{n}$ and we can apply lemma 9.5

Definition 9.7. An $x \in A$ is called an $S$-twisted coordinate, or rooted twisted coordinate, (resp. an $S$-quantum coordinate, or rooted quantum coordinate, ) if $x$ is a twisted (resp. quantum) coordinate for all $\left(A, \sigma_{n}\right)$. We call it strong if $x-\sigma_{n}(x) \in A^{\times}$for all $n \in S$.

Thus, by definition, $x$ is an $S$-quantum coordinate if and only if

$$
\forall n \in S, \quad \sigma_{n}(x)=q_{n} x+h_{n} \quad \text { with } \quad q_{n}, h_{n} \in R .
$$

When this is the case, we might also say $q$-coordinate and call $A$ a rooted quantum R-algebra, an $S$-quantum $R$-algebra or a $q$-R-algebra. We call it strong when $x$ is strong.

Lemma 9.8. Assume that $x$ is simultaneously a twisted coordinate and a rooted quantum coordinate on $A$ so that 9.1 holds. Then, $x$ is a quantum coordinate on $A$ and if we write $\sigma(x)=q x+h$ with $q, h \in R$, then $q:=\left\{q_{n}\right\}_{n \in S}$ is a system of roots of $q$. If this system is admissible, we have

$$
\forall n \in \mathbb{N}, \quad h_{n}=\left(\frac{1}{n}\right)_{q} h .
$$

Proof If we are given $m \in \mathbb{N}$ and $n \in S$, we know that $\sigma_{n}^{m}$ only depends on $r:=\frac{m}{n}$ and so does $\sigma_{n}^{m}(x)=q_{n}^{m} x+(m)_{q_{n}} h_{n}$. It immediately follows that $q$ is a system of roots. Moreover, the case $m=n$ implies that $\sigma(x)=q x+h$ with $q=q_{n}^{n}$ and $h=(n)_{q^{n}} h_{n}$ from which we derive the other assertions.

Examples 1. If we are given a system $\underline{q}$ of roots in $R$, we can endow $A:=R[x]$ or $A=R\left[x, x^{-1}\right]$ with

$$
\forall n \in S, \quad \sigma_{n}(x)=q_{n} x .
$$

In the later case, $x$ is a strong $\underline{q}$-coordinate if and only if $1-q \in R^{\times}$(and then $\underline{q}$ is also admissible). 
2. More generally, if we are given an admissible system $\underline{q}$ of roots of $q \in R$ and some $h \in R$, we can endow $R[x]$ with

$$
\forall n \in S, \quad \sigma_{n}(x)=q_{n} x+\left(\frac{1}{n}\right)_{q} h .
$$

Definition 9.9. Assume that $x$ is a $q$-coordinate on $A$ with $q$ strongly admissible. A rooted twisted differential $A$-module is an $A$-module $M$ endowed with a family of $\partial_{A, \sigma_{n}}$-derivations $\partial_{M, n}$ for all $n \in S$ such that whenever $n, n^{\prime} \in S$ with $n \mid n^{\prime}$, we have

$$
\forall s \in M, \quad \partial_{M, n}(s)=\sum_{k=1}^{n^{\prime} / n} \frac{1}{(k)_{q_{n^{\prime}}} !}\left(\prod_{i=1}^{k-1}\left(\sigma_{n}(x)-\sigma_{n^{\prime}}^{i}(x)\right)\right) \partial_{M, n^{\prime}}^{k}(s) .
$$

They form a category that we will denote by $\partial_{A, \underline{\sigma}}-$ Mod $^{\text {root }}$.

Definition 9.10. If $A$ is a $\underline{q}$ - $R$-algebra with $q$ strongly admissible, then the rooted twisted Weyl algebra of $A$ is

$$
\mathrm{D}_{A / R, \underline{\sigma}}:=\lim _{\longrightarrow} \mathrm{D}_{A / R, \sigma_{n}}
$$

with transition maps (coming from lemma 8.5 for $n, n^{\prime} \in S$ with $n \mid n^{\prime}$ given by

$$
\begin{aligned}
& \mathrm{D}_{A / R, \sigma_{n}} \longrightarrow \stackrel{\iota_{\sigma_{n^{\prime}}, n^{\prime} / n}}{\longrightarrow} \mathrm{D}_{A / R, \sigma_{n^{\prime}}} \\
& \partial_{\sigma_{n}} \longmapsto \sum_{k=1}^{n^{\prime} / n} \frac{1}{(k)_{q_{n^{\prime}}} !}\left(\prod_{i=1}^{k-1}\left(\sigma_{n}(x)-\sigma_{n^{\prime}}^{i}(x)\right)\right) \partial_{\sigma_{n^{\prime}}}^{k}
\end{aligned}
$$

Proposition 9.11. Assume that $A$ is a $q$-R-algebra with $q$ strongly admissible. Then the rooted twisted differential A-modules form an abelian category with sufficiently many injective and projective objects. Actually, if $M$ is a $\mathrm{D}_{A / R, \underline{\sigma}}$-module, then the maps

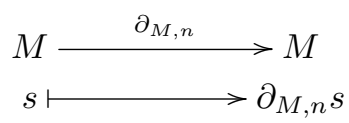

turn $M$ into a rooted twisted differential A-module and we obtain an equivalence (an isomorphism) of categories

$$
\mathrm{D}_{A / R, \underline{\sigma}}-\operatorname{Mod} \simeq \partial_{A, \underline{\sigma}}-\operatorname{Mod}^{\mathrm{root}} .
$$

Proof This is a consequence of proposition 5.6 of $[23]$.

Recall from $\left[23\right.$ that $A\left[T^{\frac{1}{S}}\right]_{\underline{\sigma}}$ denotes the non commutative Puiseux polynomial ring with the commutation rule

$$
\forall m \in \mathbb{N}, \forall n \in S, \quad T^{\frac{m}{n}} x=\sigma_{n}^{m}(x) T^{\frac{m}{n}} .
$$

One can also consider the notion of $\underline{\sigma}_{A}$-module: this is an $A$-module endowed with a compatible family of $\sigma_{A, n}$-linear endomorphisms $\sigma_{M, n}$. There exists an equivalence (an isomorphism) between the category of $A\left[T^{\frac{1}{5}}\right]_{\underline{\sigma}}$-modules and the category of $\underline{\sigma}_{A^{-}}$-modules.

Proposition 9.12. Assume that $x$ is a $\underline{q}$-coordinate on $A$ with $\underline{q}$ strongly admissible. Then there exists a unique A-linear homomorphism of rings

$$
\begin{aligned}
A\left[T^{\frac{1}{S}}\right]_{\underline{\sigma}} & \longrightarrow \mathrm{D}_{A / R, \underline{\sigma}} \\
T^{\frac{1}{n}} \longmapsto & \longrightarrow 1+\left(\sigma_{n}(x)-x\right) \partial_{\sigma_{n}}
\end{aligned}
$$

inducing a functor

$$
\partial_{A, \underline{\sigma}}-\operatorname{Mod} \rightarrow \underline{\sigma}_{A}-\operatorname{Mod} .
$$

If $x$ is a strong $\underline{q}$-coordinate, then the map 9.2 is an isomorphism and the functor 9.3 is an equivalence. 
Proof This will follow from theorem 6.13 of 23 once we know that the various maps for different $n$ 's are all compatible. More precisely, we have to check that the differential operators $1+\left(\sigma_{n}(x)-x\right) \partial_{\sigma_{n}}$ form a system of roots in $\mathrm{D}_{\underline{\sigma}}$. But this follows from proposition 8.7

Theorem 9.13 (Formal quantum confluence 2). Let $R$ be a $\mathbb{Q}$-algebra, $q$ a strongly admissible system of roots in $R$ and $A$ a $q$-R-algebra. Then, there exists a canonical $\bar{A}$-linear map

$$
\mathrm{D}_{A / R, \underline{\sigma}} \rightarrow \widehat{\mathrm{D}}_{A / R} \text {. }
$$

If $R$ is $\underline{q}$-divisible and $\underline{q}$ is infinite, then the map 8.2 has dense image.

Proof First of all, the map (8.2) is obtained by taking the direct limit of the maps (8.1) for all $\sigma_{n}$ 's. Now, we set $p_{n}:=q_{n}-\operatorname{char}(R)$ for all $n \in S$ and we apply the confluence theorem 8.3 to $\sigma_{n}$. If $p_{n}=0$, we are done. Otherwise, the theorem tells us that the bottom map in the following commutative diagram is surjective:

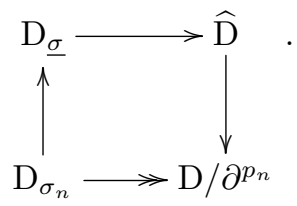

And we proved in lemma 9.5 that for all $n \in S$, we have $p_{n}>n$. Since $\underline{q}$ is infinite, we have $p_{n} \rightarrow \infty$ and we see that the image of the upper map is dense.

Corollary 9.14. Assume moreover, that $x$ is a strong q-coordinate on A. Then, the A-linear map

$$
\begin{aligned}
& A\left[T^{\frac{1}{S}}\right]_{\underline{\sigma}} \longrightarrow \widehat{\mathrm{D}}_{A / R} \\
& T^{\frac{m}{n}} \longmapsto \sum_{k=0}^{\infty} \frac{1}{k !}\left(\sigma_{n}^{m}(x)-x\right)^{k} \partial^{k}
\end{aligned}
$$

has dense image.

Proof The formula comes from corollary 7.7

Example Theorem 9.13 (and its corollary) applies in particular when $q$ is a primitive $p$-th root of unity in some algebraically closed field $K$ of characteristic zero, $q$ is a system of $p^{n}$-th roots of $q$ in $K, R$ is an algebra containing $K, A=R\left[x, x^{-1}\right]$ and $\sigma_{n}(x)=\bar{q}_{n} x$ (we should actually write $\sigma_{p^{n}}$ and $q_{p^{n}}$ but we will try to make the notations easier to use).

As we already saw, in this situation, $\mathrm{D}:=\mathrm{D}_{A / R}$ is the non commutative ring $R\left[x, x^{-1}, \partial\right]$ with the commutation rule $\partial x=x \partial+1$ and we may see $\widehat{\mathrm{D}}$ (which is not a ring) as $R\left[x, x^{-1}\right][[\partial]]$. We also want to understand the left hand side and see that $\mathrm{D}_{n}:=\mathrm{D}_{A / R, \sigma_{n}}$ is the non commutative ring $R\left[x, x^{-1}, \partial_{n}\right]$ with the commutation rule $\partial_{n} x=q_{n} x \partial_{n}+1$. The transition maps are given by the rather tricky formulas

$$
\partial_{n} \mapsto \sum_{k=1}^{p^{n^{\prime}-n}} \frac{q_{n^{\prime}}^{\frac{k(k-1)}{2}}\left(q_{n^{\prime}}-1\right)^{k-1}}{(k)_{q_{n^{\prime}}}}\left(\begin{array}{c}
p^{n^{\prime}-n}-1 \\
k-1
\end{array}\right)_{q_{n^{\prime}}} x^{k-1} \partial_{n^{\prime}}^{k},
$$

and the map of the theorem satisfies

$$
\partial_{n} \mapsto \sum_{k=1}^{\infty} \frac{1}{k !}\left(q_{n}-1\right)^{k-1} x^{k-1} \partial^{k} .
$$

Also, the map of the corollary is given by

$$
T^{r} \mapsto \sum_{k=1}^{\infty} \frac{1}{k !}\left(q^{r}-1\right)^{k} x^{k} \partial^{k}
$$

if we set $q^{m / n}:=q_{n}^{m}$. 
Remark Alexei Belov-Kanel and Maxime Konsevich proved in 5 that the Jacobian conjecture is stably equivalent to the Dixmier conjecture [14. According to the later, any endomorphism of a Weyl algebra over $\mathbb{C}$ is an automorphism. And this is still a conjecture even in dimension one. On the other hand, when $q$ is a primitive $p$-th root of unity, then the quantum Weyl algebra $D_{\sigma}$ does not satisfy the Dixmier conjecture but it is an Azumaya algebra. In particular, checking that an endomorphism is an automorphism can be done on the center. As explained by Backelin in [4], it is appealing to attack the Dixmier conjecture through quantum Weyl algebras and one can hope that theorem 9.13 might provide a tool for this quest.

\section{References}

[1] C. Raymond Adams. On the linear ordinary q-difference equation. Ann. of Math. (2), 30(14):195-205, 1928/29.

[2] Yves André. Différentielles non commutatives et théorie de Galois différentielle ou aux différences. Ann. Sci. École Norm. Sup. (4), 34(5):685-739, 2001.

[3] Yves André and Lucia Di Vizio. q-difference equations and p-adic local monodromy. Astérisque, (296):55-111, 2004. Analyse complexe, systèmes dynamiques, sommabilité des séries divergentes et théories galoisiennes. I.

[4] Erik Backelin. Endomorphisms of quantized Weyl algebras. Lett. Math. Phys., 97(3):317-338, 2011.

[5] Alexei Belov-Kanel and Maxim Kontsevich. The Jacobian conjecture is stably equivalent to the Dixmier conjecture. Mosc. Math. J., 7(2):209-218, 349, 2007.

[6] George D. Birkhoff. General theory of linear difference equations. Trans. Amer. Math. Soc., 12(2):243-284, 1911.

[7] N. Bourbaki. Éléments de mathématique. Algèbre. Chapitres 1 à 3. Hermann, Paris, 1970.

[8] N. Bourbaki. Éléments de mathématique. Algèbre. Chapitre 8. Modules et anneaux semisimples. Springer, Berlin, 2012. Second revised edition of the 1958 edition [MR0098114].

[9] R. D. Carmichael. Linear difference equations and their analytic solutions. Trans. Amer. Math. Soc., 12(1):99-134, 1911.

[10] R. D. Carmichael. The General Theory of Linear q-Difference Equations. Amer. J. Math., 34(2):147-168, 1912.

[11] Richard M. Cohn. Difference algebra. Interscience Publishers John Wiley \& Sons, New YorkLondon-Sydeny, 1965.

[12] L. Di Vizio, J.-P. Ramis, J. Sauloy, and C. Zhang. Équations aux q-différences. Gaz. Math., $(96): 20-49,2003$.

[13] Lucia Di Vizio. Introduction to $p$-adic $q$-difference equations (weak Frobenius structure and transfer theorems). In Geometric aspects of Dwork theory. Vol. I, II, pages 615-675. Walter de Gruyter GmbH \& Co. KG, Berlin, 2004.

[14] Jacques Dixmier. Sur les algèbres de Weyl. Bull. Soc. Math. France, 96:209-242, 1968.

[15] Anne Duval and Julien Roques. Familles fuchsiennes d'équations aux ( $q$-)différences et confluence. Bull. Soc. Math. France, 136(1):67-96, 2008.

[16] Michel Gros and Bernard Le Stum. Une neutralisation explicite de l'algèbre de Weyl quantique complétée. Comm. Algebra, 42(5):2163-2170, 2014. 
[17] A. Grothendieck. Éléments de géométrie algébrique. IV. Étude locale des schémas et des morphismes de schémas IV. Inst. Hautes Études Sci. Publ. Math., (32):361, 1967.

[18] C. Guichard. Sur la résolution de l'équation aux différences fines $G(x+1)-G(x)=H(x)$. Ann. Sci. École Norm. Sup. (3), 4:361-380, 1887.

[19] Charlotte Hardouin. Iterative $q$-difference Galois theory. J. Reine Angew. Math., 644:101-144, 2010.

[20] Uma N. Iyer and Timothy C. McCune. Quantum differential operators on $k[x]$. Internat. $J$. Math., 13(4):395-413, 2002.

[21] Max Karoubi and Mariano Suárez-Álvarez. Twisted Kähler differential forms. J. Pure Appl. Algebra, 181(2-3):279-289, 2003.

[22] Bernard Le Stum and Adolfo Quirós. On quantum integers and rationals. In Trends in Number Theory-Sevilla 2013, volume 649. Contemporary Math., Amer. Math. Soc., Providence, RI, 2015.

[23] Bernard Le Stum and Adolfo Quirós. Twisted calculus. Communications in Algebra, to appear, doi.org/10.1080/00927872.2018.1464168, 2018.

[24] Valery A. Lunts and Alexander L. Rosenberg. Differential operators on noncommutative rings. Selecta Math. (N.S.), 3(3):335-359, 1997.

[25] Zoghman Mebkhout and Luis Narváez Macarro. Le théorème de continuité de la division dans les anneaux d'opérateurs différentiels. J. Reine Angew. Math., 503:193-236, 1998.

[26] Andrea Pulita. p-adic confluence of $q$-difference equations. Compos. Math., 144(4):867-919, 2008.

[27] Andrea Pulita. Infinitesimal deformation of p-adic differential equations on berkovich curves. Math. Ann., 2016.

[28] Jacques Sauloy. Systèmes aux $q$-différences singuliers réguliers: classification, matrice de connexion et monodromie. Ann. Inst. Fourier (Grenoble), 50(4):1021-1071, 2000.

[29] Jacques Sauloy. Galois theory of Fuchsian $q$-difference equations. Ann. Sci. École Norm. Sup. (4), 36(6):925-968 (2004), 2003. 